\title{
A survey on the study of Hilbert-type inequalities
}

\section{Qiang Chen ${ }^{1}$ and Bicheng Yang ${ }^{2 *}$}

\section{"Correspondence:}

bcyang@gdei.edu.cn

${ }^{2}$ Department of Mathematics,

Guangdong University of

Education, Guangzhou, Guangdong 510303, P.R. China

Full list of author information is available at the end of the article

\begin{abstract}
Hilbert-type inequalities are divided three parts: Hilbert's inequalities (1908), Hardy-Hilbert-type inequalities (1934) and Yang-Hilbert-type inequalities (2009). In this paper, we give a summary of the development of the theory of Hilbert-type inequalities during the past 110 years.
\end{abstract}

MSC: 26D15; 47A07

Keywords: Hilbert-type inequality; extension; weight function; equivalent form; operator expression

\section{Hilbert's inequalities and the operator expressions}

\subsection{Hilbert's inequalities}

In 1908, Weyl [1] published the following inequality.

If $\left\{a_{m}\right\}_{m=1}^{\infty}$ and $\left\{b_{n}\right\}_{n=1}^{\infty}$ are real sequences, satisfying $0<\sum_{m=1}^{\infty} a_{m}^{2}<\infty$ and $0<\sum_{n=1}^{\infty} b_{n}^{2}<$ $\infty$, then

$$
\sum_{n=1}^{\infty} \sum_{m=1}^{\infty} \frac{a_{m} b_{n}}{m+n}<\pi\left(\sum_{m=1}^{\infty} a_{m}^{2} \sum_{n=1}^{\infty} b_{n}^{2}\right)^{\frac{1}{2}}
$$

where the constant factor $\pi$ is the best possible.

We named (1) Hilbert's inequality; it does not contain any parameter. The best possible property of the constant factor $\pi$ was proved by Schur [2] in 1911. He also gave the following integral analog of (1) at the same time.

If $f(x)$ and $g(y)$ are measurable functions, such that $0<\int_{0}^{\infty} f^{2}(x) d x<\infty$ and $0<$ $\int_{0}^{\infty} g^{2}(y) d y<\infty$, then

$$
\int_{0}^{\infty} \int_{0}^{\infty} \frac{f(x) g(y)}{x+y} d x d y<\pi\left(\int_{0}^{\infty} f^{2}(x) d x \int_{0}^{\infty} g^{2}(y) d y\right)^{\frac{1}{2}}
$$

where the constant factor $\pi$ is still the best possible.

We called (2) Hilbert's integral inequality, which still does not contain any parameter. Inequalities (1) and (2) are important in analysis and its applications. We can find a number of improvements and extensions in the vast mathematics literature, especially in [3-6]. 


\subsection{Hilbert's operators}

We may express inequality (1) by using the form of the operator as follows.

If $l^{2}$ is a space of real sequences, and $T: l^{2} \rightarrow l^{2}$ is a linear operator, for any $a=\left\{a_{m}\right\}_{m=1}^{\infty} \in$ $l^{2}$, there exists a $c=\left\{c_{n}\right\}_{n=1}^{\infty} \in l^{2}$, satisfying

$$
c_{n}=(T a)(n)=\sum_{m=1}^{\infty} \frac{a_{m}}{m+n}, \quad n \in \mathbf{N}=\{1,2, \ldots\} .
$$

Hence for any $b=\left\{b_{n}\right\}_{n=1}^{\infty} \in l^{2}$, we may write the inner product of $T a$ and $b$ as follows:

$$
(T a, b)=(c, b)=\sum_{n=1}^{\infty}\left(\sum_{m=1}^{\infty} \frac{a_{m}}{m+n}\right) b_{n}=\sum_{n=1}^{\infty} \sum_{m=1}^{\infty} \frac{a_{m} b_{n}}{m+n} .
$$

Expressing the norm of $a$ as $\|a\|_{2}=\left\{\sum_{n=1}^{\infty} a_{n}^{2}\right\}^{1 / 2}$, in view of (4), inequality (1) may be rewritten as follows:

$$
(T a, b)<\pi\|a\|_{2}\|b\|_{2},
$$

where $\|a\|_{2},\|b\|_{2}>0$. We may prove that $T$ is a bounded operator and obtain the norm $\|T\|=\pi(c f$. [7]). We call $T$ Hilbert's operator.

For $\|a\|_{2}>0$, the equivalent form of (5) is given as $\|T a\|_{2}<\pi\|a\|_{2}$, e.t.

$$
\sum_{n=1}^{\infty}\left(\sum_{m=1}^{\infty} \frac{a_{m}}{m+n}\right)^{2}<\pi^{2} \sum_{m=1}^{\infty} a_{m}^{2}
$$

where the constant factor $\pi^{2}$ is still the best possible. Obviously, inequalities (6) and (1) are equivalent $(c f .[3])$.

Similarly, if $L^{2}\left(\mathbf{R}_{+}\right)$is a space of real functions, we may define Hilbert's integral operator $\widetilde{T}: L^{2}\left(\mathbf{R}_{+}\right) \rightarrow L^{2}\left(\mathbf{R}_{+}\right)$as follows.

For any $f \in L^{2}\left(\mathbf{R}_{+}\right)$, there exists a $h=\widetilde{T} f \in L^{2}\left(\mathbf{R}_{+}\right)$, satisfying

$$
(\widetilde{T} f)(y)=h(y)=\int_{0}^{\infty} \frac{f(x)}{x+y} d x, \quad y \in(0, \infty) .
$$

Hence, for any $g \in L^{2}\left(\mathbf{R}_{+}\right)$, we may still can indicate the inner product of $\widetilde{T} f$ and $g$ as follows:

$$
(\widetilde{T} f, g)=\int_{0}^{\infty}\left(\int_{0}^{\infty} \frac{f(x)}{x+y} d x\right) g(y) d y=\int_{0}^{\infty} \int_{0}^{\infty} \frac{f(x) g(y)}{x+y} d x d y .
$$

Setting the norm of $f$ as $\|f\|_{2}=\left(\int_{0}^{\infty} f^{2}(x) d x\right)^{\frac{1}{2}}$, if $\|f\|_{2},\|g\|_{2}>0$, then (2) may be rewritten as follows:

$$
(\widetilde{T} f, g)<\pi\|f\|_{2}\|g\|_{2} .
$$

It follows that $\|\widetilde{T} f\|=\pi$ (cf. [8]), and then we have the equivalent form of (2) as $\|\widetilde{T} f\|_{2}<$ $\pi\|f \mid\|_{2} \|$, e.t. (cf. [3]):

$$
\int_{0}^{\infty}\left(\int_{0}^{\infty} \frac{f(x)}{x+y} d x\right)^{2} d y<\pi^{2} \int_{0}^{\infty} f^{2}(x) d x
$$


where the constant factor $\pi^{2}$ is still the best possible. It is obvious that inequality (10) is the integral analog of (6).

\subsection{A more accurate discrete Hilbert's inequality}

If we let the subscripts $m, n$ of the double series go from 0 to infinity, then we may rewrite inequality (1) equivalently in the following form:

$$
\sum_{n=0}^{\infty} \sum_{m=0}^{\infty} \frac{a_{m} b_{n}}{m+n+2}<\pi\left(\sum_{m=0}^{\infty} a_{m}^{2} \sum_{n=0}^{\infty} b_{n}^{2}\right)^{\frac{1}{2}}
$$

where the constant factor $\pi$ is still the best possible. Obviously, we may raise the following question:

Is there a positive constant $\alpha(<2)$ that leaves the inequality still valid as we replace 2 by $\alpha$ in the kernel $\frac{1}{m+n+2}$ ? The answer is positive. That is, we have the following more accurate Hilbert inequality (for short, Hilbert's inequality) (cf. [3]):

$$
\sum_{n=0}^{\infty} \sum_{m=0}^{\infty} \frac{a_{m} b_{n}}{m+n+1}<\pi\left(\sum_{m=0}^{\infty} a_{m}^{2} \sum_{n=0}^{\infty} b_{n}^{2}\right)^{\frac{1}{2}},
$$

where the constant factor $\pi$ is the best possible.

Since for $a_{m}, b_{n} \geq 0, \alpha \geq 1$,

$$
\sum_{n=0}^{\infty} \sum_{m=0}^{\infty} \frac{a_{m} b_{n}}{m+n+\alpha} \leq \sum_{n=0}^{\infty} \sum_{m=0}^{\infty} \frac{a_{m} b_{n}}{m+n+1},
$$

then by (12) and for $\alpha \geq 1$, we have

$$
\sum_{n=0}^{\infty} \sum_{m=0}^{\infty} \frac{a_{m} b_{n}}{m+n+\alpha}<\pi\left(\sum_{m=0}^{\infty} a_{m}^{2} \sum_{n=0}^{\infty} b_{n}^{2}\right)^{\frac{1}{2}} .
$$

For $1 \leq \alpha<2$, inequality (13) is a refinement of (11). Obviously, we have a refinement of (6), which is equivalent to (13) as follows:

$$
\sum_{n=0}^{\infty}\left(\sum_{m=0}^{\infty} \frac{a_{m}}{m+n+\alpha}\right)^{2}<\pi^{2} \sum_{m=0}^{\infty} a_{m}^{2} \quad(1 \leq \alpha<2) .
$$

For $0<\alpha<1$, in 1936, Ingham [9] gave the following.

If $\alpha \geq \frac{1}{2}$, then

$$
\sum_{n=0}^{\infty} \sum_{m=0}^{\infty} \frac{a_{m} a_{n}}{m+n+\alpha} \leq \pi \sum_{m=0}^{\infty} a_{m}^{2}
$$

if $0<\alpha<\frac{1}{2}$, then

$$
\sum_{n=0}^{\infty} \sum_{m=0}^{\infty} \frac{a_{m} a_{n}}{m+n+\alpha} \leq \frac{\pi}{\sin (\alpha \pi)} \sum_{m=0}^{\infty} a_{m}^{2}
$$


Note that if we set $x=X+\frac{\alpha}{2}, y=Y+\frac{\alpha}{2}, F(X)=f\left(X+\frac{\alpha}{2}\right)$ and $G(Y)=g\left(Y+\frac{\alpha}{2}\right)(\alpha \in \mathbf{R}=$ $(-\infty, \infty))$ in $(2)$, then we obtain

$$
\int_{-\frac{\alpha}{2}}^{\infty} \int_{-\frac{\alpha}{2}}^{\infty} \frac{F(X) G(Y)}{X+Y+\alpha} d X d Y<\pi\left(\int_{-\frac{\alpha}{2}}^{\infty} F^{2}(X) d X \int_{-\frac{\alpha}{2}}^{\infty} G^{2}(Y) d Y\right)^{\frac{1}{2}} .
$$

It is said that for $\alpha \geq \frac{1}{2}$, inequality (17) is an integral analog of (13) (for $G=F$ ) and for $0<\alpha<\frac{1}{2}$, (17) is not an integral analog of (13), since the two constant factors are different. In recent years, by using the improved Euler-Maclaurin summation formula and introducing parameters, a few authors gave some more accurate Hilbert-type inequalities as (13) (cf. [10-17]).

\section{Hardy-Hilbert-type inequalities with a pair of conjugate exponents}

\subsection{Hardy-Hilbert's inequalities and the operator expressions}

In 1925, by introducing one pair of conjugate exponents $(p, q)$ with $\frac{1}{p}+\frac{1}{q}=1$, Hardy [18] gave an extension of (1) as follows.

If $p>1, a_{m}, b_{n} \geq 0$, such that $0<\sum_{m=1}^{\infty} a_{m}^{p}<\infty$ and $0<\sum_{n=1}^{\infty} b_{n}^{q}<\infty$, then

$$
\sum_{n=1}^{\infty} \sum_{m=1}^{\infty} \frac{a_{m} b_{n}}{m+n}<\frac{\pi}{\sin \left(\frac{\pi}{p}\right)}\left(\sum_{m=1}^{\infty} a_{m}^{p}\right)^{\frac{1}{p}}\left(\sum_{n=1}^{\infty} b_{n}^{q}\right)^{\frac{1}{q}},
$$

where the constant factor $\frac{\pi}{\sin (\pi / p)}$ is the best possible.

The equivalent form of (18) is as follows:

$$
\sum_{n=1}^{\infty}\left(\sum_{m=1}^{\infty} \frac{a_{m}}{m+n}\right)^{p}<\left[\frac{\pi}{\sin \left(\frac{\pi}{p}\right)}\right]^{p} \sum_{m=1}^{\infty} a_{m}^{p}
$$

where the constant factor $\left[\frac{\pi}{\sin (\pi / p)}\right]^{p}$ is still the best possible.

In the same way, inequalities (12) and (14) (for $\alpha=1$ ) may be extended to the following equivalent forms (cf. [3]):

$$
\begin{aligned}
& \sum_{n=0}^{\infty} \sum_{m=0}^{\infty} \frac{a_{m} b_{n}}{m+n+1}<\frac{\pi}{\sin \left(\frac{\pi}{p}\right)}\left(\sum_{m=0}^{\infty} a_{m}^{p}\right)^{\frac{1}{p}}\left(\sum_{n=0}^{\infty} b_{n}^{q}\right)^{\frac{1}{q}}, \\
& \sum_{n=0}^{\infty}\left(\sum_{m=0}^{\infty} \frac{a_{m}}{m+n+1}\right)^{p}<\left[\frac{\pi}{\sin \left(\frac{\pi}{p}\right)}\right]^{p} \sum_{m=0}^{\infty} a_{m}^{p},
\end{aligned}
$$

where the constant factors $\frac{\pi}{\sin (\pi / p)}$ and $\left[\frac{\pi}{\sin (\pi / p)}\right]^{p}$ are the best possible. The equivalent integral analogs of (18) and (19) are given as follows:

$$
\begin{aligned}
& \int_{0}^{\infty} \int_{0}^{\infty} \frac{f(x) g(y)}{x+y} d x d y<\frac{\pi}{\sin \left(\frac{\pi}{p}\right)}\left(\int_{0}^{\infty} f^{p}(x) d x\right)^{\frac{1}{p}}\left(\int_{0}^{\infty} g^{q}(y) d y\right)^{\frac{1}{q}} \\
& \int_{0}^{\infty}\left(\int_{0}^{\infty} \frac{f(x)}{x+y} d x\right)^{p} d y<\left[\frac{\pi}{\sin \left(\frac{\pi}{p}\right)}\right]^{p} \int_{0}^{\infty} f^{p}(x) d x
\end{aligned}
$$

with the best possible constant factors. We call (18) Hardy-Hilbert's inequality and call (22) Hardy-Hilbert's integral inequality. 
Inequality (20) may be expressed in the form of the operator as follows.

If $l^{p}$ is a space of real sequences, $T_{p}: l^{p} \rightarrow l^{p}$ is a linear operator, such that for any nonnegative sequence $a=\left\{a_{m}\right\}_{m=1} \in l^{p}$, there exists a $T_{p} a=c=\left\{c_{n}\right\}_{n=1}^{\infty} \in l^{p}$, satisfying

$$
c_{n}=\left(T_{p} a\right)(n)=\sum_{m=0}^{\infty} \frac{a_{m}}{m+n+1}, \quad n \in \mathbf{N}_{0}=\mathbf{N} \cup\{0\} .
$$

For any non-negative sequence $b=\left\{b_{n}\right\}_{n=1} \in l^{q}$, we can indicate the formal inner product of $T_{p} a$ and $b$ as follows:

$$
\left(T_{p} a, b\right)=\sum_{n=0}^{\infty}\left(\sum_{m=0}^{\infty} \frac{a_{m}}{m+n+1}\right) b_{n}=\sum_{n=0}^{\infty} \sum_{m=0}^{\infty} \frac{a_{m} b_{n}}{m+n+1} .
$$

Setting the norm of $a$ as $\|a\|_{p}=\left(\sum_{n=0}^{\infty}\left|a_{n}\right|^{p}\right)^{\frac{1}{p}}$, then inequality (20) may be rewritten as follows:

$$
\left(T_{p} a, b\right)<\frac{\pi}{\sin \left(\frac{\pi}{p}\right)}\|a\|_{p}\|b\|_{q}
$$

where $\|a\|_{p},\|b\|_{q}>0$. We call $T_{p}$ Hardy-Hilbert's operator.

Similarly, if $L^{p}\left(\mathbf{R}_{+}\right)$is a space of real functions, we may define the following HardyHilbert's integral operator $\widetilde{T}_{p}: L^{p}\left(\mathbf{R}_{+}\right) \rightarrow L^{p}\left(\mathbf{R}_{+}\right)$as follows.

For any $f(\geq 0) \in L^{p}\left(\mathbf{R}_{+}\right)$, there exists a $h=\widetilde{T}_{p} f \in L^{p}\left(\mathbf{R}_{+}\right)$, satisfying

$$
\left(\widetilde{T}_{p} f\right)(y)=h(y)=\int_{0}^{\infty} \frac{f(x)}{x+y} d x, \quad y \in \mathbf{R}_{+} .
$$

For any $g(\geq 0) \in L^{q}\left(\mathbf{R}_{+}\right)$, we can indicate the formal inner product of $\widetilde{T}_{p} f$ and $g$ as follows:

$$
\left(\widetilde{T}_{p} f, g\right)=\int_{0}^{\infty} \int_{0}^{\infty} \frac{f(x) g(y)}{x+y} d x d y .
$$

Setting the norm of $f$ as $\|f\|_{p}=\left(\int_{0}^{\infty}|f(x)|^{p} d x\right)^{\frac{1}{p}}$, then inequality (22) may be rewritten as follows:

$$
\left(\widetilde{T}_{p} f, g\right)<\frac{\pi}{\sin \left(\frac{\pi}{p}\right)}\|f\|_{p}\|g\|_{q} .
$$

\subsection{Some kinds of Hardy-Hilbert-type inequalities}

(1) For $(p, q)$ not being a pair of conjugate exponents, we have the following results (cf. [3], Theorem 339).

$$
\begin{aligned}
& \text { If } p>1, q>1, \frac{1}{p}+\frac{1}{q} \geq 1,0<\lambda=2-\left(\frac{1}{p}+\frac{1}{q}\right) \leq 1 \text {, then } \\
& \sum_{n=1}^{\infty} \sum_{m=1}^{\infty} \frac{a_{m} b_{n}}{(m+n)^{\lambda}} \leq K\left(\sum_{m=1}^{\infty} a_{m}^{p}\right)^{\frac{1}{p}}\left(\sum_{n=1}^{\infty} b_{n}^{q}\right)^{\frac{1}{q}},
\end{aligned}
$$

where $K=K(p, q)$ relates to $p, q$, only for $\frac{1}{p}+\frac{1}{q}=1, \lambda=2-\left(\frac{1}{p}+\frac{1}{q}\right)=1$, the constant factor $K$ is the best possible. 
The integral analogs of (30) are given as follows:

$$
\int_{0}^{\infty} \int_{0}^{\infty} \frac{f(x) g(y)}{(x+y)^{\lambda}} d x d y \leq K\left(\int_{0}^{\infty} f^{p}(x) d x\right)^{\frac{1}{p}}\left(\int_{0}^{\infty} g^{q}(y) d y\right)^{\frac{1}{q}}
$$

We also find an extension of (31) as follows (cf. [4]).

$$
\begin{aligned}
& \text { If } p>1, q>1, \frac{1}{p}+\frac{1}{q}>1,0<\lambda=2-\left(\frac{1}{p}+\frac{1}{q}\right)<1 \text {, then } \\
& \qquad \int_{-\infty}^{\infty} \int_{-\infty}^{\infty} \frac{f(x) g(y)}{|x+y|^{\lambda}} d x d y \leq k(p, q)\left(\int_{-\infty}^{\infty} f^{p}(x) d x\right)^{\frac{1}{p}}\left(\int_{-\infty}^{\infty} g^{q}(y) d y\right)^{\frac{1}{q}} .
\end{aligned}
$$

For $f(x)=g(x)=0, x \in(-\infty, 0]$, inequality (32) reduces to (31). Levin [19] also studied the expression forms of the constant factors in (30) and (31). But he did not prove their best possible property. In 1951, Bonsall [20] considered the case of (31) as regards the general kernel.

(2) If $p>1, \frac{1}{p}+\frac{1}{q}=1, h(t)>0, \phi(s)=\int_{0}^{\infty} h(t) t^{s-1} d t \in \mathbf{R}_{+}, f(x), g(y) \geq 0$, then we have the following integral inequalities with the non-homogeneous kernel (cf. [3], Theorem 350):

$$
\begin{aligned}
& \int_{0}^{\infty} \int_{0}^{\infty} h(x y) f(x) g(y) d x d y \\
& <\phi\left(\frac{1}{p}\right)\left(\int_{0}^{\infty} x^{p-2} f^{p}(x) d x\right)^{\frac{1}{p}}\left(\int_{0}^{\infty} g^{q}(y) d y\right)^{\frac{1}{q}}, \\
& \int_{0}^{\infty}\left(\int_{0}^{\infty} h(x y) f(x) d x\right)^{p} d y<\phi^{p}\left(\frac{1}{p}\right) \int_{0}^{\infty} x^{p-2} f^{p}(x) d x, \\
& \int_{0}^{\infty}\left(\int_{0}^{\infty} h(x y) f(x) d x\right)^{p} d y<\phi^{p}\left(\frac{1}{p}\right) \int_{0}^{\infty} x^{p-2} f^{p}(x) d x, \\
& \int_{0}^{\infty} y^{p-2}\left(\int_{0}^{\infty} h(x y) f(x) d x\right)^{p} d y<\phi^{p}\left(\frac{1}{q}\right) \int_{0}^{\infty} f^{p}(x) d x,
\end{aligned}
$$

where the integrals on the right-hand side are positive. The authors did not proved that the above constant factors are the best possible.

(3) If $p>1, \frac{1}{p}+\frac{1}{q}=1, h(t)>0$ is decreasing with respect to $t>0, \phi(s)=\int_{0}^{\infty} h(t) t^{s-1} d t \in$ $\mathbf{R}_{+}, f(x), a_{n} \geq 0$, then we have the following half-discrete inequalities ( $c f$. [3], Theorem 351):

$$
\begin{aligned}
& \int_{0}^{\infty}\left(\sum_{n=1}^{\infty} h(n x) a_{n}\right)^{p} d x<\phi^{p}\left(\frac{1}{p}\right) \sum_{n=1}^{\infty} n^{p-2} a_{n}^{p}, \\
& \sum_{n=1}^{\infty}\left(\int_{0}^{\infty} h(n x) f(x) d x\right)^{p}<\phi^{p}\left(\frac{1}{p}\right) \int_{0}^{\infty} x^{p-2} f^{p}(x) d x, \\
& \int_{0}^{\infty} x^{p-2}\left(\sum_{n=1}^{\infty} h(n x) a_{n}\right)^{p} d x<\phi^{p}\left(\frac{1}{q}\right) \sum_{n=1}^{\infty} a_{n}^{p}, \\
& \sum_{n=1}^{\infty} n^{p-2}\left(\int_{0}^{\infty} h(n x) f(x) d x\right)^{p}<\phi^{p}\left(\frac{1}{q}\right) \int_{0}^{\infty} f^{p}(x) d x,
\end{aligned}
$$

where the integrals and series on the right-hand side are positive. The authors also did not prove that the above constant factors are the best possible. 


\subsection{Hardy-Hilbert-type inequalities with the general homogeneous kernel of degree -1}

If $\alpha \in \mathbf{R}$, the function $k(x, y)$ is measurable in $\mathbf{R}_{+}^{2}$, satisfying for any $x, y, u>0, k(u x, u y)=$ $u^{\alpha} k(x, y)$, then we call $k(x, y)$ the homogeneous function of degree $\alpha$.

In 1934, Hardy et al. published the following theorem (cf. [3], Theorem 318 and Theorem 319).

Suppose that $p>1, \frac{1}{p}+\frac{1}{q}=1, k_{1}(x, y)(\geq 0)$ is a homogeneous function of degree -1 in $\mathbf{R}_{+}^{2}$. If $k_{p}=\int_{0}^{\infty} k_{1}(u, 1) u^{-\frac{1}{p}} d u$ is finite, then we have $k_{p}=\int_{0}^{\infty} k_{1}(1, u) u^{-\frac{1}{q}} d u$ and the following equivalent inequalities:

$$
\begin{aligned}
& \int_{0}^{\infty} \int_{0}^{\infty} k_{1}(x, y) f(x) g(y) d x d y \\
& \quad \leq k_{p}\left(\int_{0}^{\infty} f^{p}(x) d x\right)^{\frac{1}{p}}\left(\int_{0}^{\infty} g^{q}(y) d y\right)^{\frac{1}{q}}, \\
& \int_{0}^{\infty}\left(\int_{0}^{\infty} k_{1}(x, y) f(x) d x\right)^{p} d y \leq k_{p}^{p} \int_{0}^{\infty} f^{p}(x) d x,
\end{aligned}
$$

where the constant $k_{p}$ is the best possible.

Moreover, if both $k_{1}(u, 1) u^{\frac{-1}{p}}$ and $k_{1}(1, u) u^{\frac{-1}{q}}$ are decreasing in $\mathbf{R}_{+}$, then we have the following equivalent forms:

$$
\begin{aligned}
& \sum_{n=1}^{\infty} \sum_{m=1}^{\infty} k_{1}(m, n) a_{m} b_{n} \leq k_{p}\left(\sum_{m=1}^{\infty} a_{m}^{p}\right)^{\frac{1}{p}}\left(\sum_{n=1}^{\infty} b_{n}^{q}\right)^{\frac{1}{q}} \\
& \sum_{n=1}^{\infty}\left(\sum_{m=1}^{\infty} k_{1}(m, n) a_{m}\right)^{p} \leq k_{p}^{p} \sum_{n=1}^{\infty} a_{n}^{p} .
\end{aligned}
$$

For $0<p<1$, if $k_{p}$ is finite, then we have the reverses of (41) and (42). (Note that we have not seen any proof of (41) and (42), and the reverse examples in the book [3].)

We name $k_{1}(x, y)$ the kernel of (41) and (42). If all the integrals and series in the righthand side of inequalities (41)-(44) are positive, then we can get the following particular examples (cf. [3]):

(i) For $k_{1}(x, y)=\frac{1}{x+y},(41)-(44)$ deduce to (22), (23), (18), and (19).

(ii) For $k_{1}(x, y)=\frac{1}{\max \{x, y\}}$, (41)-(44) deduce to the following two pairs of equivalent forms:

$$
\begin{aligned}
& \int_{0}^{\infty} \int_{0}^{\infty} \frac{f(x) g(y)}{\max \{x, y\}} d x d y<p q\left(\int_{0}^{\infty} f^{p}(x) d x\right)^{\frac{1}{p}}\left(\int_{0}^{\infty} g^{q}(y) d y\right)^{\frac{1}{q}}, \\
& \int_{0}^{\infty}\left(\int_{0}^{\infty} \frac{f(x)}{\max \{x, y\}} d x\right)^{p} d y<(p q)^{p} \int_{0}^{\infty} f^{p}(x) d x \\
& \sum_{n=1}^{\infty} \sum_{m=1}^{\infty} \frac{a_{m} b_{n}}{\max \{m, n\}}<p q\left(\sum_{m=1}^{\infty} a_{m}^{p}\right)^{\frac{1}{p}}\left(\sum_{n=1}^{\infty} b_{n}^{q}\right)^{\frac{1}{q}}, \\
& \sum_{n=1}^{\infty}\left(\sum_{m=1}^{\infty} \frac{a_{m}}{\max \{m, n\}}\right)^{p}<(p q)^{p} \sum_{n=1}^{\infty} a_{n}^{p} .
\end{aligned}
$$


(iii) For $k_{1}(x, y)=\frac{\ln (x / y)}{x-y},(41)-(44)$ deduce to the following two pairs of equivalent forms:

$$
\begin{aligned}
& \int_{0}^{\infty} \int_{0}^{\infty} \frac{\ln \left(\frac{x}{y}\right) f(x) g(y)}{x-y} d x d y \\
& <\left[\frac{\pi}{\sin \left(\frac{\pi}{p}\right)}\right]^{2}\left(\int_{0}^{\infty} f^{p}(x) d x\right)^{\frac{1}{p}}\left(\int_{0}^{\infty} g^{q}(y) d y\right)^{\frac{1}{q}}, \\
& \int_{0}^{\infty}\left(\int_{0}^{\infty} \frac{\ln \left(\frac{x}{y}\right) f(x)}{x-y} d x\right)^{p} d y<\left[\frac{\pi}{\sin \left(\frac{\pi}{p}\right)}\right]^{2 p} \int_{0}^{\infty} f^{p}(x) d x \\
& \sum_{n=1}^{\infty} \sum_{m=1}^{\infty} \frac{\ln \left(\frac{m}{n}\right) a_{m} b_{n}}{m-n}<\left[\frac{\pi}{\sin \left(\frac{\pi}{p}\right)}\right]^{2}\left(\sum_{m=1}^{\infty} a_{m}^{p}\right)^{\frac{1}{p}}\left(\sum_{n=1}^{\infty} b_{n}^{q}\right)^{\frac{1}{q}} \\
& \sum_{n=1}^{\infty}\left(\sum_{m=1}^{\infty} \frac{\ln \left(\frac{m}{n}\right) a_{m}}{m-n}\right)^{p}<\left[\frac{\pi}{\sin \left(\frac{\pi}{p}\right)}\right]^{2 p} \sum_{n=1}^{\infty} a_{n}^{p} .
\end{aligned}
$$

Note that the constant factors in the above inequalities are all the best possible. We call (47) and (51) Hardy-Littlewood-Polya's inequalities, or H-L-P inequalities. We find that the kernels in the above inequalities are all decreasing. But this is not necessary. For example, we find the following two pairs of equivalent forms with the non-decreasing kernel (cf. [21, 22]):

$$
\begin{aligned}
& \int_{0}^{\infty} \int_{0}^{\infty} \frac{\left|\ln \left(\frac{x}{y}\right)\right| f(x) g(y)}{\max \{x, y\}} d x d y \\
& <\left(p^{2}+q^{2}\right)\left(\int_{0}^{\infty} f^{p}(x) d x\right)^{\frac{1}{p}}\left(\int_{0}^{\infty} g^{q}(y) d y\right)^{\frac{1}{q}} \\
& \int_{0}^{\infty}\left(\int_{0}^{\infty} \frac{\left|\ln \left(\frac{x}{y}\right)\right| f(x)}{\max \{x, y\}} d x\right)^{p} d y<\left(p^{2}+q^{2}\right)^{p} \int_{0}^{\infty} f^{p}(x) d x \\
& \sum_{n=1}^{\infty} \sum_{m=1}^{\infty} \frac{\left|\ln \left(\frac{m}{n}\right)\right| a_{m} b_{n}}{\max \{m, n\}}<\left(p^{2}+q^{2}\right)\left(\sum_{m=1}^{\infty} a_{m}^{p}\right)^{\frac{1}{p}}\left(\sum_{n=1}^{\infty} b_{n}^{q}\right)^{\frac{1}{q}}, \\
& \sum_{n=1}^{\infty}\left(\sum_{m=1}^{\infty} \frac{\left|\ln \left(\frac{m}{n}\right)\right| a_{m}}{\max \{m, n\}}\right)^{p}<\left(p^{2}+q^{2}\right)^{p} \sum_{n=1}^{\infty} a_{n}^{p},
\end{aligned}
$$

where the constant factors $p^{2}+q^{2}$ and $\left(p^{2}+q^{2}\right)^{p}$ are the best possible.

Other inequalities of this type with the best constants are provided as follows ( $c f$. [23, 24]):

$$
\begin{aligned}
& \int_{0}^{\infty} \int_{0}^{\infty} \frac{\left|\ln \left(\frac{x}{y}\right)\right| f(x) g(y)}{x+y} d x d y \\
& <c_{0}(p)\left(\int_{0}^{\infty} f^{p}(x) d x\right)^{\frac{1}{p}}\left(\int_{0}^{\infty} g^{p}(y) d y\right)^{\frac{1}{q}} \\
& \int_{0}^{\infty}\left(\int_{0}^{\infty} \frac{\left|\ln \left(\frac{x}{y}\right)\right| f(x)}{x+y} d x\right)^{p} d y<c_{0}^{p}(p) \int_{0}^{\infty} f^{p}(x) d x \\
& \sum_{n=1}^{\infty} \sum_{m=1}^{\infty} \frac{\left|\ln \left(\frac{m}{n}\right)\right| a_{m} b_{n}}{m+n}<c_{0}(2)\left(\sum_{m=1}^{\infty} a_{m}^{2} \sum_{n=1}^{\infty} b_{n}^{2}\right)^{\frac{1}{2}}
\end{aligned}
$$




$$
\sum_{n=1}^{\infty}\left(\sum_{m=1}^{\infty} \frac{\left|\ln \left(\frac{m}{n}\right)\right| a_{m}}{m+n}\right)^{2}<c_{0}^{2}(2) \sum_{n=1}^{\infty} a_{n}^{2}
$$

where the constant factor $c_{0}(p)$ is indicated by

$$
c_{0}(p)=2 \sum_{n=1}^{\infty}(-1)^{n-1}\left[\frac{1}{\left(n-\frac{1}{p}\right)^{2}}-\frac{1}{\left(n-\frac{1}{q}\right)^{2}}\right] .
$$

\subsection{Two multiple Hardy-Hilbert-type inequalities with the homogeneous kernels of degree $-n+1$}

Suppose $n \in \mathbf{N} \backslash\{1\}, n$ numbers $p, q, \ldots, r$ satisfying $p, q, \ldots, r>1, p^{-1}+q^{-1}+\cdots+r^{-1}=1$, $k(x, y, \ldots, z) \geq 0$ is a homogeneous function of degree $-n+1$. If

$$
k=\int_{0}^{\infty} \int_{0}^{\infty} \cdots \int_{0}^{\infty} k(1, y, \ldots, z) y^{-\frac{1}{q}} \cdots z^{-\frac{1}{r}} d y \cdots d z
$$

is a finite number, $f, g, \ldots, h$ are non-negative measurable functions in $\mathbf{R}_{+}$, then we have the following multiple Hilbert-type integral inequality (cf. [3], Theorem 322):

$$
\begin{aligned}
& \int_{0}^{\infty} \int_{0}^{\infty} \cdots \int_{0}^{\infty} k(x, y, \ldots, z) f(x) g(y) \cdots h(z) d x d y \cdots d z \\
& \quad \leq k\left(\int_{0}^{\infty} f^{p}(x) d x\right)^{\frac{1}{p}}\left(\int_{0}^{\infty} g^{q}(y) d y\right)^{\frac{1}{q}} \cdots\left(\int_{0}^{\infty} h^{r}(z) d z\right)^{\frac{1}{r}} .
\end{aligned}
$$

Moreover, if $a_{m}, b_{n}, \ldots, c_{s} \geq 0, k(1, y, \ldots, z) x^{0} y^{-\frac{1}{q}} \cdots z^{-\frac{1}{r}}, k(x, 1, \ldots, z) \times x^{-\frac{1}{p}} y^{0} \cdots z^{-\frac{1}{r}}, \ldots$, $k(x, y, \ldots, 1) x^{-\frac{1}{p}} y^{-\frac{1}{q}} \cdots z^{0}$ are all decreasing with respect to any single variable in $\mathbf{R}_{+}$, then we have

$$
\begin{aligned}
& \sum_{s=1}^{\infty} \cdots \sum_{n=1}^{\infty} \sum_{m=1}^{\infty} k(m, n, \ldots, s) a_{m} b_{n} \cdots c_{s} \\
& \leq k\left(\sum_{m=1}^{\infty} a_{m}^{p}\right)^{\frac{1}{p}}\left(\sum_{n=1}^{\infty} b_{n}^{q}\right)^{\frac{1}{q}} \cdots\left(\sum_{s=1}^{\infty} c_{s}^{r}\right)^{\frac{1}{r}} .
\end{aligned}
$$

For $n=2$, inequalities (61) and (62) reduce, respectively, to (41) and (43).

\section{Modern research for Hilbert's inequalities and Hardy-Hilbert's inequalities}

\subsection{Modern research for Hilbert's integral inequality}

(1) In 1979, based on an improvement of Hölder's inequality, $\mathrm{Hu}$ [25] gave a refinement of

(2) (for $f=g$ ) as follows:

$$
\begin{aligned}
& \int_{0}^{\infty} \int_{0}^{\infty} \frac{f(x) f(y)}{x+y} d x d y \\
& \quad<\pi\left[\left(\int_{0}^{\infty} f^{2}(x) d x\right)^{2}-\frac{1}{4}\left(\int_{0}^{\infty} f^{2}(x) \cos \sqrt{x} d x\right)^{2}\right]^{\frac{1}{2}} .
\end{aligned}
$$

Since then, he published many interesting results similar to (63) (cf. [6]). 
(2) In 1998, Pachpatte [26] gave an inequality similar to (2) as follows.

For $a, b>0$,

$$
\begin{aligned}
& \int_{0}^{a} \int_{0}^{b} \frac{f(x) g(y)}{x+y} d x d y \\
& \quad<\frac{\sqrt{a b}}{2}\left[\int_{0}^{a}(a-x) f^{\prime 2}(x) d x \int_{0}^{b}(b-y) g^{\prime 2}(y) d y\right]^{\frac{1}{2}} .
\end{aligned}
$$

Some improvements and extensions were made by Zhao et al. [27-29]. We can find other work of Pachpatte in [30].

(3) In 1998, by introducing parameters $\lambda \in(0,1]$ and $a, b \in \mathbf{R}_{+}(a<b)$, Yang [31] gave an extension of (2) as follows:

$$
\begin{aligned}
& \int_{a}^{b} \int_{a}^{b} \frac{f(x) g(y)}{(x+y)^{\lambda}} d x d y \\
& \quad<B\left(\frac{\lambda}{2}, \frac{\lambda}{2}\right)\left[1-\left(\frac{a}{b}\right)^{\frac{\lambda}{4}}\right]\left(\int_{a}^{b} x^{1-\lambda} f^{2}(x) d x \int_{a}^{b} y^{1-\lambda} g^{2}(y) d y\right)^{\frac{1}{2}}
\end{aligned}
$$

where $B(u, v)$ is the beta function. In 1999, Kuang [32] gave another extension of (2) as follows.

$$
\begin{aligned}
& \text { For } \lambda \in\left(\frac{1}{2}, 1\right] \\
& \qquad \int_{0}^{\infty} \int_{0}^{\infty} \frac{f(x) f(y)}{x^{\lambda}+y^{\lambda}} d x d y<\frac{\pi}{\lambda \sin \left(\frac{\pi}{2 \lambda}\right)}\left(\int_{0}^{\infty} f^{2}(x) d x \int_{0}^{\infty} g^{2}(y) d y\right)^{\frac{1}{2}} .
\end{aligned}
$$

We can find other work of Kuang in [5] and [33].

(4) In 1999, by using the methods of algebra and analysis, Gao [34] gave an improvement of (2) as follows:

$$
\int_{0}^{\infty} \int_{0}^{\infty} \frac{f(x) f(y)}{x+y} d x d y<\pi \sqrt{1-R}\left(\int_{0}^{\infty} f^{2}(x) d x \int_{0}^{\infty} g^{2}(y) d y\right)^{\frac{1}{2}}
$$

where $R=\frac{1}{\pi}\left(\frac{u}{\|g\|}-\frac{v}{\|f\|}\right)^{2}, u=\sqrt{\frac{2}{\pi}}(g, e), v=\sqrt{2 \pi}\left(f, e^{-x}\right), e(y)=\int_{0}^{\infty} \frac{e^{x}}{x+y} d x$. We can find other work of Gao and Hsu in [35].

(5) In 2002, by using the operator theory, Zhang [36] gave an improvement of (2) as follows:

$$
\begin{aligned}
& \int_{0}^{\infty} \int_{0}^{\infty} \frac{f(x) g(y)}{x+y} d x d y \\
& \quad \leq \frac{\pi}{\sqrt{2}}\left[\int_{0}^{\infty} f^{2}(x) d x \int_{0}^{\infty} g^{2}(y) d y+\left(\int_{0}^{\infty} f(x) g(x) d x\right)^{2}\right]^{\frac{1}{2}} .
\end{aligned}
$$

\subsection{On the way of weight coefficients for giving a strengthened version of Hilbert's inequality}

In 1991, for giving an improvement of (1), Hsu and Wang [37] raised the way of weight coefficient as follows. 
At first, by using Cauchy's inequality in the left-hand side of (1), it follows:

$$
\begin{aligned}
I & =\sum_{n=1}^{\infty} \sum_{m=1}^{\infty} \frac{a_{m} b_{n}}{m+n}=\sum_{n=1}^{\infty} \sum_{m=1}^{\infty} \frac{1}{m+n}\left[\left(\frac{m}{n}\right)^{\frac{1}{4}} a_{m}\right]\left[\left(\frac{n}{m}\right)^{\frac{1}{4}} b_{n}\right] \\
& \leq\left\{\sum_{m=1}^{\infty}\left[\sum_{n=1}^{\infty} \frac{1}{m+n}\left(\frac{m}{n}\right)^{\frac{1}{2}}\right] a_{m}^{2} \sum_{n=1}^{\infty}\left[\sum_{m=1}^{\infty} \frac{1}{m+n}\left(\frac{m}{n}\right)^{\frac{1}{2}}\right] b_{n}^{2}\right\}^{\frac{1}{2}} .
\end{aligned}
$$

Then define the weight coefficient

$$
\omega(n):=\sum_{m=1}^{\infty} \frac{1}{m+n}\left(\frac{m}{n}\right)^{\frac{1}{2}}, \quad n \in \mathbf{N},
$$

and rewrite (69) as follows:

$$
I \leq\left(\sum_{m=1}^{\infty} \omega(m) a_{m}^{2} \sum_{n=1}^{\infty} \omega(n) b_{n}^{2}\right)^{\frac{1}{2}}
$$

Afterwards, setting

$$
\omega(n)=\pi-\frac{\theta(n)}{n^{1 / 2}}, \quad n \in \mathbf{N},
$$

where $\theta(n)=(\pi-\omega(n)) n^{1 / 2}$, and estimating the series of $\theta(n)$, it follows that

$$
\theta(n)=\left[\pi-\sum_{m=1}^{\infty} \frac{1}{m+n}\left(\frac{m}{n}\right)^{\frac{1}{2}}\right] n^{1 / 2}>\theta=1.1213^{+} .
$$

Then by (72), it yields

$$
\omega(n)<\pi-\frac{\theta}{n^{1 / 2}}, \quad n \in \mathbf{N}, \theta=1.1213^{+} .
$$

In view of (71), a strengthened version of (1) is given as follows:

$$
I<\left[\sum_{m=1}^{\infty}\left(\pi-\frac{\theta}{m^{1 / 2}}\right) a_{m}^{2} \sum_{n=1}^{\infty}\left(\pi-\frac{\theta}{n^{1 / 2}}\right) b_{n}^{2}\right]^{\frac{1}{2}} .
$$

Hsu also raised the open problem of obtaining the best value of (75). In 1992, Gao [38] gave the best value $\theta_{0}=1.281669^{+}$.

Still in 1991, by using the above method, a strengthened version of (8) was given by [39] as follows:

$$
\begin{aligned}
I< & \left\{\sum_{m=1}^{\infty}\left[\frac{\pi}{\sin \left(\frac{\pi}{p}\right)}-\frac{p-1}{m^{1 / p}+m^{-1 / q}}\right] a_{m}^{p}\right\}^{\frac{1}{p}} \\
& \times\left\{\sum_{n=1}^{\infty}\left[\frac{\pi}{\sin \left(\frac{\pi}{p}\right)}-\frac{q-1}{n^{1 / q}+n^{-1 / p}}\right] b_{n}^{q}\right\}^{\frac{1}{q}} .
\end{aligned}
$$


In 1997, by using the method of weight coefficients and the improved Euler-Maclaurin summation formula, Yang and Gao [40] gave

$$
I<\left\{\sum_{m=1}^{\infty}\left[\frac{\pi}{\sin \left(\frac{\pi}{p}\right)}-\frac{1-\gamma}{m^{1 / p}}\right] a_{m}^{p}\right\}^{\frac{1}{p}}\left\{\sum_{n=1}^{\infty}\left[\frac{\pi}{\sin \left(\frac{\pi}{p}\right)}-\frac{1-\gamma}{n^{1 / q}}\right] b_{n}^{q}\right\}^{\frac{1}{q}}
$$

where $1-\gamma=0.42278433^{+}$( $\gamma$ is the Euler constant). We can find similar work in Gao and Yang [41].

In 1998, Yang and Debnath [42] gave another, strengthened, version of (8), which is an improvement of (76). We can find some strengthened versions of (12) and (20) in [43-45].

\subsection{Hilbert's inequalities and Hardy-Hilbert's inequalities with independent parameters}

In 1998, by using the optimized weight coefficients and introducing an independent parameter $\lambda \in(0,1]$, Yang [31] gave an extension of (2) as follows.

$$
\begin{aligned}
& \text { If } 0<\int_{0}^{\infty} x^{1-\lambda} f^{2}(x) d x<\infty \text { and } 0<\int_{0}^{\infty} y^{1-\lambda} g^{2}(y) d y<\infty \text {, then } \\
& \int_{0}^{\infty} \int_{0}^{\infty} \frac{f(x) g(y)}{(x+y)^{\lambda}} d x d y \\
& \quad<B\left(\frac{\lambda}{2}, \frac{\lambda}{2}\right)\left(\int_{0}^{\infty} x^{1-\lambda} f^{2}(x) d x \int_{0}^{\infty} y^{1-\lambda} g^{2}(y) d y\right)^{\frac{1}{2}}
\end{aligned}
$$

where the constant factor $B\left(\frac{\lambda}{2}, \frac{\lambda}{2}\right)$ is the best possible. The proof about the best possible property of the constant factor was given by [46], and the expressions of the beta function $B(u, v)$ are given in Wang and Guo [47]:

$$
\begin{aligned}
B(u, v) & =\int_{0}^{\infty} \frac{t^{u-1} d t}{(1+t)^{u+v}}=\int_{0}^{1}(1-t)^{u-1} t^{\nu-1} d t \\
& =\int_{1}^{\infty} \frac{(t-1)^{u-1} d t}{t^{u+v}} \quad(u, v>0) .
\end{aligned}
$$

Some extensions of (18), (20), and (22) were given by [48-50] as follows.

If $\lambda>2-\min \{p, q\}$, then

$$
\begin{aligned}
& \int_{0}^{\infty} \int_{0}^{\infty} \frac{f(x) g(y)}{(x+y)^{\lambda}} d x d y \\
& \quad<B\left(\frac{p+\lambda-2}{p}, \frac{q+\lambda-2}{q}\right)\left(\int_{0}^{\infty} x^{1-\lambda} f^{p}(x) d x\right)^{\frac{1}{p}}\left(\int_{0}^{\infty} y^{1-\lambda} g^{q}(y) d y\right)^{\frac{1}{q}}
\end{aligned}
$$

if $2-\min \{p, q\}<\lambda \leq 2$, then

$$
\begin{aligned}
& \sum_{n=1}^{\infty} \sum_{m=1}^{\infty} \frac{a_{m} b_{n}}{(m+n)^{\lambda}} \\
& \quad<B\left(\frac{p+\lambda-2}{p}, \frac{q+\lambda-2}{q}\right)\left(\sum_{m=1}^{\infty} m^{1-\lambda} a_{m}^{p}\right)^{\frac{1}{p}}\left(\sum_{n=1}^{\infty} n^{1-\lambda} b_{n}^{q}\right)^{\frac{1}{q}},
\end{aligned}
$$




$$
\begin{aligned}
& \sum_{n=0}^{\infty} \sum_{m=0}^{\infty} \frac{a_{m} b_{n}}{(m+n+1)^{\lambda}} \\
& \quad<B\left(\frac{p+\lambda-2}{p}, \frac{q+\lambda-2}{q}\right)\left[\sum_{m=0}^{\infty}\left(m+\frac{1}{2}\right)^{1-\lambda} a_{m}^{p}\right]^{\frac{1}{p}}\left[\sum_{n=0}^{\infty}\left(n+\frac{1}{2}\right)^{1-\lambda} b_{n}^{q}\right]^{\frac{1}{q}},
\end{aligned}
$$

where the constant factor $B\left(\frac{p+\lambda-2}{p}, \frac{q+\lambda-2}{q}\right)$ is the best possible.

Yang [51] also proved that (81) is valid for $p=2$ and $\lambda \in(0,4]$. Yang [52, 53] gave another extensions of (18) and (20) as follows.

If $0<\lambda \leq \min \{p, q\}$, then

$$
\sum_{n=1}^{\infty} \sum_{m=1}^{\infty} \frac{a_{m} b_{n}}{m^{\lambda}+n^{\lambda}}<\frac{\pi}{\lambda \sin \left(\frac{\pi}{p}\right)}\left[\sum_{m=1}^{\infty} m^{(p-1)(1-\lambda)} a_{m}^{p}\right]^{\frac{1}{p}}\left[\sum_{n=1}^{\infty} n^{(q-1)(1-\lambda)} b_{n}^{q}\right]^{\frac{1}{q}}
$$

if $0<\lambda \leq 1$, then

$$
\begin{aligned}
& \sum_{n=0}^{\infty} \sum_{m=0}^{\infty} \frac{a_{m} b_{n}}{\left(m+\frac{1}{2}\right)^{\lambda}+\left(n+\frac{1}{2}\right)^{\lambda}} \\
& <\frac{\pi}{\lambda \sin \left(\frac{\pi}{p}\right)}\left[\sum_{m=0}^{\infty}\left(m+\frac{1}{2}\right)^{p-1-\lambda} a_{m}^{p}\right]^{\frac{1}{p}}\left[\sum_{n=0}^{\infty}\left(n+\frac{1}{2}\right)^{q-1-\lambda} b_{n}^{q}\right]^{\frac{1}{q}} .
\end{aligned}
$$

In 2004, Yang [54] discovered the following dual form of (18):

$$
\sum_{n=1}^{\infty} \sum_{m=1}^{\infty} \frac{a_{m} b_{n}}{m+n}<\frac{\pi}{\sin \left(\frac{\pi}{p}\right)}\left(\sum_{m=1}^{\infty} m^{p-2} a_{m}^{p}\right)^{\frac{1}{p}}\left(\sum_{n=1}^{\infty} n^{q-2} b_{n}^{q}\right)^{\frac{1}{q}} .
$$

Inequality (85) is similar to (18) but different and for $p=2$, both of them reduce to (1).

For $\lambda=1$, (84) reduces to the dual form of (20) as follows:

$$
\sum_{n=0}^{\infty} \sum_{m=0}^{\infty} \frac{a_{m} b_{n}}{m+n+1}<\frac{\pi}{\sin \left(\frac{\pi}{p}\right)}\left[\sum_{m=0}^{\infty}\left(m+\frac{1}{2}\right)^{p-2} a_{m}^{p}\right]^{\frac{1}{p}}\left[\sum_{n=0}^{\infty}\left(n+\frac{1}{2}\right)^{q-2} b_{n}^{q}\right]^{\frac{1}{q}} .
$$

We can find some best extensions of the H-L-P inequalities such as (37)-(48) in [55-61], by introducing some independent parameters.

In 2001, by introducing some parameters, Hong [62] gave a multiple integral inequality, which is an extension of (18). He et al. [63] gave a similar result for particular conjugate exponents. For making an improvement of their work, Yang [64] gave the following inequality, which is a best extension of (18).

If $n \in \mathbf{N} \backslash\{1\}, p_{i}>1, \sum_{i=1}^{n} \frac{1}{p_{i}}=1, \lambda>n-\min _{1 \leq i \leq n}\left\{p_{i}\right\}, f_{i}(t) \geq 0$, and $0<\int_{0}^{\infty} t^{n-1-\lambda} f_{i}^{p_{i}}(t) d t<$ $\infty(i=1,2, \ldots, n)$, then we have

$$
\begin{aligned}
& \int_{0}^{\infty} \cdots \int_{0}^{\infty} \frac{\prod_{i=1}^{n} f_{i}\left(x_{i}\right)}{\left(\sum_{i=1}^{n} x_{i}\right)^{\lambda}} d x_{1} \cdots d x_{n} \\
& <\frac{1}{\Gamma(\lambda)} \prod_{i=1}^{n}\left(\frac{p_{i}+\lambda-n}{p_{i}}\right)\left(\int_{0}^{\infty} t^{n-1-\lambda} f_{i}^{p_{i}}(t) d t\right)^{\frac{1}{p_{i}}},
\end{aligned}
$$


where the constant factor $\frac{1}{\Gamma(\lambda)} \prod_{i=1}^{n}\left(\frac{p_{i}+\lambda-n}{p_{i}}\right)$ is the best possible. In particular, for $\lambda=n-1$, it follows that

$$
\begin{aligned}
& \int_{0}^{\infty} \cdots \int_{0}^{\infty} \frac{\prod_{i=1}^{n} f_{i}\left(x_{i}\right)}{\left(\sum_{i=1}^{n} x_{i}\right)^{n-1}} d x_{1} \cdots d x_{n} \\
& <\frac{1}{(n-2) !} \prod_{i=1}^{n}\left(1-\frac{1}{p_{i}}\right)\left(\int_{0}^{\infty} f_{i}^{p_{i}}(t) d t\right)^{\frac{1}{p_{i}}} .
\end{aligned}
$$

In 2003, Yang and Rassias [65] introduced the method of weight coefficients and considered its applications to Hilbert-type inequalities. They summarized how to use the method of weight coefficients to obtain some new improvements and generalizations of the Hilbert-type inequalities. Since then, a number of authors discussed this problem ( $c f$. [66-86]). But how to give a best extension of inequalities (85) and (18) was solved in 2004 by introducing two pairs of conjugate exponents.

\subsection{Hilbert-type inequalities with two conjugate exponents and multi-parameters}

In 2004, by introducing an independent parameter $\lambda>0$ and two pairs of conjugate exponents $(p, q)$ and $(r, s)$ with $\frac{1}{p}+\frac{1}{q}=\frac{1}{r}+\frac{1}{s}=1$, Yang [87] gave an extension of (2) as follows.

If $p, r>1$, and the integrals of the right-hand side are positive, then

$$
\begin{aligned}
& \int_{0}^{\infty} \int_{0}^{\infty} \frac{f(x) g(y)}{x^{\lambda}+y^{\lambda}} d x d y \\
& <\frac{\pi}{\lambda \sin \left(\frac{\pi}{r}\right)}\left[\int_{0}^{\infty} x^{p\left(1-\frac{\lambda}{r}\right)-1} f^{p}(x) d x\right]^{\frac{1}{p}}\left[\int_{0}^{\infty} y^{q\left(1-\frac{\lambda}{s}\right)-1} g^{q}(y) d y\right]^{\frac{1}{q}},
\end{aligned}
$$

where the constant factor $\frac{\pi}{\lambda \sin \left(\frac{\pi}{r}\right)}$ is the best possible.

For $\lambda=1, r=q, s=p$, inequality (89) reduces to (22); for $\lambda=1, r=p, s=q$, inequality (89) reduces to the dual form of (22) as follows:

$$
\begin{aligned}
& \int_{0}^{\infty} \int_{0}^{\infty} \frac{f(x) g(y)}{x+y} d x d y \\
& \quad<\frac{\pi}{\sin \left(\frac{\pi}{p}\right)}\left(\int_{0}^{\infty} x^{p-2} f^{p}(x) d x\right)^{\frac{1}{p}}\left(\int_{0}^{\infty} y^{q-2} g^{q}(y) d y\right)^{\frac{1}{q}} .
\end{aligned}
$$

In 2005, by introducing an independent parameter $\lambda>0$, and two pairs of generalized conjugate exponents $\left(p_{1}, p_{2}, \ldots, p_{n}\right)$ and $\left(r_{1}, r_{2}, \ldots, r_{n}\right)$ with $\sum_{i=1}^{n} \frac{1}{p_{i}}=\sum_{i=1}^{n} \frac{1}{r_{i}}=1$, Yang et al. [88] gave a multiple integral inequality as follows.

For $p_{i}, r_{i}>1(i=1,2, \ldots, n)$,

$$
\begin{aligned}
& \int_{0}^{\infty} \cdots \int_{0}^{\infty} \frac{\prod_{i=1}^{n} f_{i}\left(x_{i}\right)}{\left(\sum_{i=1}^{n} x_{i}\right)^{\lambda}} d x_{1} \cdots d x_{n} \\
& <\frac{1}{\Gamma(\lambda)} \prod_{i=1}^{n}\left(\frac{\lambda}{r_{i}}\right)\left[\int_{0}^{\infty} t^{p_{i}\left(1-\frac{\lambda}{r_{i}}\right)-1} f_{i}^{p_{i}}(t) d t\right]^{\frac{1}{p_{i}}},
\end{aligned}
$$

where the constant factor $\frac{1}{\Gamma(\lambda)} \prod_{i=1}^{n}\left(\frac{\lambda}{r_{i}}\right)$ is the best possible. For $r_{i}=\frac{p_{i} \lambda}{p_{i}-\lambda-n}(i=1,2, \ldots, n)$, inequality (91) reduces to (87); for $n=2, p_{1}=p, p_{2}=q, r_{1}=r$, and $r_{2}=s$, inequality (91) 
reduces to the following:

$$
\begin{aligned}
& \int_{0}^{\infty} \int_{0}^{\infty} \frac{f(x) g(y)}{(x+y)^{\lambda}} d x d y \\
& \quad<B\left(\frac{\lambda}{r}, \frac{\lambda}{s}\right)\left[\int_{0}^{\infty} x^{p\left(1-\frac{\lambda}{r}\right)-1} f^{p}(x) d x\right]^{\frac{1}{p}}\left[\int_{0}^{\infty} y^{q\left(1-\frac{\lambda}{s}\right)-1} g^{q}(y) d y\right]^{\frac{1}{q}} .
\end{aligned}
$$

It is obvious that inequality (92) is another best extension of (22).

In 2006, by using two pairs of conjugate exponents $(p, q)$ and $(r, s)$ with $p, r>1$, Hong [89] gave a multi-variable integral inequality as follows.

If $\mathbf{R}_{+}^{n}=\left\{x=\left(x_{1}, x_{2}, \ldots, x_{n}\right) ; x_{i}>0, i=1,2, \ldots, n\right\}, \alpha, \beta, \lambda>0,\|x\|_{\alpha}=\left(\sum_{i=1}^{n} x_{i}^{\alpha}\right)^{\frac{1}{\alpha}}, f, g \geq 0$, $0<\int_{\mathbf{R}_{+}^{n}}\|x\|_{\alpha}^{p\left(n-\frac{\beta \lambda}{r}\right)-n} f^{p}(x) d x<\infty$ and $0<\int_{\mathbf{R}_{+}^{n}}\|y\|_{\alpha}^{q\left(n-\frac{\beta \lambda}{s}\right)-n} g^{q}(y) d y<\infty$, then

$$
\begin{gathered}
\int_{\mathbf{R}_{+}^{n}} \int_{\mathbf{R}_{+}^{n}} \frac{f(x) g(y) d x d y}{\left(\|x\|_{\alpha}^{\beta}+\|y\|_{\alpha}^{\beta}\right)^{\lambda}} \\
<\frac{\Gamma^{n}\left(\frac{1}{\alpha}\right)}{\beta \alpha^{n-1} \Gamma\left(\frac{n}{\alpha}\right)} B\left(\frac{\lambda}{r}, \frac{\lambda}{s}\right)\left[\int_{\mathbf{R}_{+}^{n}}\|x\|_{\alpha}^{p\left(n-\frac{\beta \lambda}{r}\right)-n} f^{p}(x) d x\right]^{\frac{1}{p}} \\
\quad \times\left[\int_{\mathbf{R}_{+}^{n}}\|y\|_{\alpha}^{q\left(n-\frac{\beta \lambda}{s}\right)-n} g^{q}(y) d y\right]^{\frac{1}{q}},
\end{gathered}
$$

where the constant factor $\frac{\Gamma^{n}\left(\frac{1}{\alpha}\right)}{\beta \alpha^{n-1} \Gamma\left(\frac{n}{\alpha}\right)} B\left(\frac{\lambda}{r}, \frac{\lambda}{s}\right)$ is the best possible. In particular, for $n=1$, (93) reduces to Hong's work in [90]; for $n=\beta=1$, (93) reduces to (92). In 2007, Zhong and Yang [91] generalized (93) to a general homogeneous kernel and proposed the reversion. Some other results on the multi-dimensional Hilbert-type inequalities are provided by [92-95].

We can find another inequality with two parameters as follows (cf. [96]):

$$
\sum_{n=1}^{\infty} \sum_{m=1}^{\infty} \frac{a_{m} b_{n}}{\left(m^{\alpha}+n^{\alpha}\right)^{\lambda}}<\frac{1}{\alpha} B\left(\frac{\lambda}{r}, \frac{\lambda}{s}\right)\left[\sum_{m=1}^{\infty} m^{p\left(1-\frac{\alpha \lambda}{r}\right)-1} a_{m}^{p}\right]^{\frac{1}{p}}\left[\sum_{n=1}^{\infty} n^{q\left(1-\frac{\alpha \lambda}{s}\right)-1} b_{n}^{q}\right]^{\frac{1}{q}},
$$

where $\alpha, \lambda>0, \alpha \lambda \leq \min \{r, s\}$. In particular, for $\alpha=1$, we have

$$
\sum_{n=1}^{\infty} \sum_{m=1}^{\infty} \frac{a_{m} b_{n}}{(m+n)^{\lambda}}<B\left(\frac{\lambda}{r}, \frac{\lambda}{s}\right)\left[\sum_{m=1}^{\infty} m^{p\left(1-\frac{\lambda}{r}\right)-1} a_{m}^{p}\right]^{\frac{1}{p}}\left[\sum_{n=1}^{\infty} n^{q\left(1-\frac{\lambda}{s}\right)-1} b_{n}^{q}\right]^{\frac{1}{q}} .
$$

For $\lambda=1, r=q$, (95) reduces to (18), and for $\lambda=1, r=p$, (95) reduces to (85). Some other results are provided by [97-99].

Also we can see the reverse form as follows (cf. [100]):

$$
\sum_{n=0}^{\infty} \sum_{m=0}^{\infty} \frac{a_{m} b_{n}}{(m+n+1)^{2}}>2\left\{\sum_{m=0}^{\infty}\left[1-\frac{1}{4(m+1)^{2}}\right] \frac{a_{m}^{p}}{2 m+1}\right\}^{\frac{1}{p}}\left\{\sum_{n=0}^{\infty} \frac{b_{n}^{q}}{2 n+1}\right\}^{\frac{1}{q}},
$$

where $0<p<1, \frac{1}{p}+\frac{1}{q}=1$. The other results on the reverse of the Hilbert-type inequalities are found in [101-107]. 
In 2006, Xin [108] gave a best extension of H-L-P integral inequality (41) as follows:

$$
\begin{aligned}
& \int_{0}^{\infty} \int_{0}^{\infty} \frac{\ln \left(\frac{x}{y}\right)}{x^{\lambda}-y^{\lambda}} f(x) g(y) d x d y \\
& \quad<\left[\frac{\pi}{\sin \left(\frac{\pi}{r}\right)}\right]^{2}\left[\int_{0}^{\infty} x^{p\left(1-\frac{\lambda}{r}\right)-1} f^{p}(x) d x\right]^{\frac{1}{p}}\left[\int_{0}^{\infty} y^{q\left(1-\frac{\lambda}{s}\right)-1} g^{q}(y) d y\right]^{\frac{1}{q}} .
\end{aligned}
$$

Zhong and Yang [109] gave an extension of another H-L-P integral inequality (37) as follows:

$$
\begin{aligned}
& \int_{0}^{\infty} \int_{0}^{\infty} \frac{f(x) g(y)}{\max \left\{x^{\lambda}, y^{\lambda}\right\}} d x d y \\
& \quad<\frac{r s}{\lambda}\left[\int_{0}^{\infty} x^{p\left(1-\frac{\lambda}{r}\right)-1} f^{p}(x) d x\right]^{\frac{1}{p}}\left[\int_{0}^{\infty} y^{q\left(1-\frac{\lambda}{s}\right)-1} g^{q}(y) d y\right]^{\frac{1}{q}}
\end{aligned}
$$

Zhong and Yang [110] also gave the reverse form of (98).

Considering a particular kernel, Yang [111] gave

$$
\begin{aligned}
& \sum_{n=1}^{\infty} \sum_{m=1}^{\infty} \frac{a_{m} b_{n}}{(\sqrt{m}+\sqrt{n}) \sqrt{\max \{m, n\}}} \\
& <4 \ln 2\left(\sum_{m=1}^{\infty} m^{\frac{p}{2}-1} a_{m}^{p}\right)^{\frac{1}{p}}\left(\sum_{n=1}^{\infty} n^{\frac{q}{2}-1} b_{n}^{q}\right)^{\frac{1}{q}}
\end{aligned}
$$

He also gave (cf. [112])

$$
\begin{aligned}
& \sum_{n=1}^{\infty} \sum_{m=1}^{\infty} \frac{a_{m} b_{n}}{(m+a n)^{2}+n^{2}} \\
& \quad<\left(\frac{\pi}{2}-\arctan a\right)\left(\sum_{m=1}^{\infty} \frac{a_{m}^{p}}{m}\right)^{\frac{1}{p}}\left(\sum_{n=1}^{\infty} \frac{b_{n}^{q}}{n}\right)^{\frac{1}{q}} \quad(a \geq 0) .
\end{aligned}
$$

By using residue theory, Yang [113] obtained

$$
\begin{aligned}
& \int_{0}^{\infty} \int_{0}^{\infty} \frac{f(x) g(y)}{(x+a y)(x+b y)(x+c y)} d x d y \\
& \quad<k\left(\int_{0}^{\infty} x^{-\frac{p}{2}-1} f^{p}(x) d x\right)^{\frac{1}{p}}\left(\int_{0}^{\infty} y^{-\frac{q}{2}-1} g^{q}(y) d y\right)^{\frac{1}{q}}
\end{aligned}
$$

where $k=\frac{1}{(\sqrt{a}+\sqrt{b})(\sqrt{b}+\sqrt{c})(\sqrt{a}+\sqrt{c})}(a, b, c>0)$.

The constant factors in the above new inequalities are all the best possible. We can find some other new work in [114-120].

In 2005, Yang [121] gave a half-discrete inequality with the kernel $\frac{1}{(1+n x)^{\lambda}}$ by introducing a variable and proved that the constant factor is the best possible. In 2011, Yang [122] deduced a half-discrete Hardy-Hilbert inequality with the best possible constant factor 
$B\left(\lambda_{1}, \lambda_{2}\right):$

$$
\begin{aligned}
& \int_{0}^{\infty} \sum_{n=1}^{\infty} \frac{a_{n} f(x)}{(x+n)^{\lambda}} d x \\
& <B\left(\lambda_{1}, \lambda_{2}\right)\left[\int_{0}^{\infty} x^{p\left(1-\lambda_{1}\right)-1} f^{p}(x) d x\right]^{\frac{1}{p}}\left[\sum_{n=1}^{\infty} n^{q\left(1-\lambda_{2}\right)-1} a_{n}^{q}\right]^{\frac{1}{q}},
\end{aligned}
$$

where $\lambda_{1}>0,0<\lambda_{2} \leq 1, \lambda_{1}+\lambda_{2}=\lambda$.

Zhong et al. [123, 124] investigated several half-discrete Hilbert-type inequalities. A half-discrete Hilbert-type inequality with a general homogeneous kernel $k_{\lambda}(x, n)$ of degree $-\lambda \in \mathbf{R}$ and a best constant factor $k\left(\lambda_{1}\right)$ was obtained, which is an extension of (102) $(c f .[125])$. Also a half-discrete Hilbert-type inequality with a general non-homogeneous kernel $k_{\lambda}(1, x n)$ and a best constant factor was given by Yang [126].

\subsection{Modern research for Hilbert-type operators}

Suppose that $H$ is a separable Hilbert space and $T: H \rightarrow H$ is a bounded self-adjoint semipositive definite operator. In 2002, Zhang [36] gave the following inequality:

$$
(a, T b)^{2} \leq \frac{\|T\|^{2}}{2}\left(\|a\|^{2}\|b\|^{2}+(a, b)^{2}\right) \quad(a, b \in H)
$$

where $(a, b)$ is the inner product of $a$ and $b$, and $\|a\|=\sqrt{(a, a)}$ is the norm of $a$. Since the Hilbert integral operator $\widetilde{T}$ defined by (7) satisfies the condition of (103) with $\|\widetilde{T}\|=\pi$, inequality (2) may be improved as (68). Since the operator $T_{p}$ defined by (24) (for $p=q=2$ ) satisfies the condition of (103) (cf. [7]), we may improve (12) to the following form:

$$
\sum_{n=0}^{\infty} \sum_{m=0}^{\infty} \frac{a_{m} b_{n}}{m+n+1}<\frac{\pi}{\sqrt{2}}\left[\sum_{m=0}^{\infty} a_{m}^{2} \sum_{n=0}^{\infty} b_{n}^{2}+\left(\sum_{n=0}^{\infty} a_{n} b_{n}\right)^{2}\right]^{\frac{1}{2}} .
$$

The key of applying (103) is to obtain the norm of the operator and to show the property of semi-definite. Now, we consider the concept and the properties of Hilbert-type integral operator as follows.

Suppose that $p>1, \frac{1}{p}+\frac{1}{q}=1, L^{r}\left(\mathbf{R}_{+}\right)(r=p, q)$ are real normal linear spaces and $k(x, y)$ is a non-negative symmetric measurable function in $\mathbf{R}_{+}^{2}$ satisfying

$$
\int_{0}^{\infty} k(x, t)\left(\frac{x}{t}\right)^{\frac{1}{r}} d t=k_{0}(p) \in \mathbf{R} \quad(x>0) .
$$

We define an integral operator as

$$
T: L^{r}\left(\mathbf{R}_{+}\right) \rightarrow L^{r}\left(\mathbf{R}_{+}\right) \quad(r=p, q),
$$

for any $f(\geq 0) \in L^{p}\left(\mathbf{R}_{+}\right)$, there exists a $h=T f \in L^{p}\left(\mathbf{R}_{+}\right)$, such that

$$
(T f)(y)=h(y)=\int_{0}^{\infty} k(x, y) f(x) d x \quad(y>0)
$$


or for any $g(\geq 0) \in L^{q}\left(\mathbf{R}_{+}\right)$, there exists a $\tilde{h}=T g \in L^{q}\left(\mathbf{R}_{+}\right)$, such that

$$
(T g)(x)=\tilde{h}(x)=\int_{0}^{\infty} k(x, y) g(y) d y \quad(x>0) .
$$

In 2006, Yang [127] proved that the operator $T$ defined by (105) or (106) are bounded with $\|T\| \leq k_{0}(p)$. The following are some results in this paper.

If $\varepsilon>0$, is small enough and the integral $\int_{0}^{\infty} k(x, t)\left(\frac{x}{t}\right)^{\frac{1+\varepsilon}{r}} d t(r=p, q ; x>0)$ is convergent to a constant $k_{\varepsilon}(p)$ independent of $x$ satisfying $k_{\varepsilon}(p)=k_{0}(p)+o(1)\left(\varepsilon \rightarrow 0^{+}\right)$, then $\|T\|=k_{0}(p)$. If $\|T\|>0, f \in L^{p}\left(\mathbf{R}_{+}\right), g \in L^{q}\left(\mathbf{R}_{+}\right),\|f\|_{p},\|g\|_{q}>0$, then we have the following equivalent inequalities:

$$
\begin{aligned}
& (T f, g)<\|T\| \cdot\|f\|_{p}\|g\|_{q}, \\
& \|T f\|_{p}<\|T\| \cdot\|f\|_{p} .
\end{aligned}
$$

Some particular cases are considered in this paper.

Yang [128] also considered some properties of Hilbert-type integral operator (for $p=$ $q=2$ ). For the homogeneous kernel of degree -1 , Yang [129] considered some sufficient conditions to obtain $\|T\|=k_{0}(p)$. We can find some properties of the discrete Hilbert-type operator in the disperse space in Yang [130-133]. A multiple integral operator is scored by Bényi and Oh [134]. In 2009, Yang [135] summarized the above part results. Some other works about Hilbert-type operators and inequalities with the general homogeneous kernel and multi-parameters were provided by [136-145].

During 2009-2014, Yang published six books about the theory of Hilbert-type operators with their norms and inequalities. On January of 2009, Yang's first book about the integral and discrete Hilbert-type operators with the general homogeneous kernels of nonnegative number degree and two pairs of conjugate exponents as well as the related inequalities was published by Chinese Science Press (cf. [146]). On October of 2009, Yang's second book about Hilbert-type integral operators with the general homogeneous kernels of real number degree and two pairs of conjugate exponents as well as their inequalities was published by Bentham Science Publishers Ltd. (cf. [147]). On February of 2011, Yang's third book about discrete Hilbert-type operators as well as the related inequalities with the same kernels and parameters in integrals was published by Bentham Science Publishers Ltd. (cf. [148]). In 2012-2013, Yang published two books that considered multiple half-discrete Hilbert-type operators and their inequalities (cf. $[149,150])$. In 2014, Yang and Debnath published a book considering general half-discrete operators and their inequalities. These six books provide an extensive account of these types of operators and inequalities successfully.

\section{Yang-Hilbert-type inequalities with two pairs of conjugate exponents and independent parameters}

\subsection{Yang-Hilbert-type integral inequalities}

In 2009, Yang [147] (Theorem 5.1.6) gave an extension of (91) as follows.

If $n \in \mathbf{N} \backslash\{1\}, p_{i}>1, r_{i} \neq 0(i=1,2, \ldots, n), \sum_{i=1}^{n} \frac{1}{p_{i}}=\sum_{i=1}^{n} \frac{1}{r_{i}}=1, \lambda \in \mathbf{R}, k_{\lambda}\left(x_{1}, \ldots, x_{n}\right)(\geq 0)$ is a homogeneous function of degree $-\lambda$ in $\mathbf{R}_{+}^{n}$,

$$
k_{\lambda}\left(r_{1}, \ldots, r_{n-1}\right)=\int_{0}^{\infty} \cdots \int_{0}^{\infty} k_{\lambda}\left(u_{1}, \ldots, u_{n-1}, 1\right) \prod_{j=1}^{n-1} u_{j}^{\frac{\lambda}{r_{j}}-1} d u_{1} \cdots d u_{n-1} \in \mathbf{R}_{+},
$$


$f_{i}(t) \geq 0$ and $0<\int_{0}^{\infty} t^{p_{i}\left(1-\frac{\lambda}{r_{i}}\right)-1} f_{i}^{p_{i}}(t) d t<\infty(i=1,2, \ldots, n)$, then we have the following inequality:

$$
\begin{gathered}
\int_{0}^{\infty} \cdots \int_{0}^{\infty} k_{\lambda}\left(x_{1}, \ldots, x_{n}\right) \prod_{i=1}^{n} f_{i}\left(x_{i}\right) d x_{1} \cdots d x_{n} \\
<k_{\lambda}\left(r_{1}, \ldots, r_{n-1}\right)\left[\int_{0}^{\infty} t^{p_{i}\left(1-\frac{\lambda}{r_{i}}\right)-1} f_{i}^{p_{i}}(t) d t\right]^{\frac{1}{p_{i}}},
\end{gathered}
$$

where the constant factor $k_{\lambda}\left(r_{1}, \ldots, r_{n-1}\right)$ is the best possible.

In this reference, the equivalent form of (109), the reverses, the operator expressions, and some particular examples are provided.

In Theorem 6.14 of this book, Yang also gave the following multi-dimensional integral inequalities, an extension of (93).

If $\lambda \in \mathbf{R}, p>1, r, s \neq 0, \frac{1}{p}+\frac{1}{q}=\frac{1}{r}+\frac{1}{s}=1, k_{\lambda}(x, y)(\geq 0)$ is a homogeneous function of degree $-\lambda$ in $\mathbf{R}_{+}^{2}$,

$$
k_{\lambda}(r)=\int_{0}^{\infty} k_{\lambda}(u, 1) u^{\frac{\lambda}{r}-1} d u \in \mathbf{R}_{+},
$$

$x, y \in \mathbf{R}_{+}^{n}, \alpha>0, f, g \geq 0,0<\int_{\mathbf{R}_{+}^{n}}\|x\|_{\alpha}^{p\left(n-\frac{\lambda}{r}\right)-n} f^{p}(x) d x<\infty$, and $0<\int_{\mathbf{R}_{+}^{n}}\|x\|_{\alpha}^{q\left(n-\frac{\lambda}{s}\right)-n} g^{q}(x) d x<$ $\infty$, then we have the following inequality:

$$
\begin{gathered}
\int_{\mathbf{R}_{+}^{n}} \int_{\mathbf{R}_{+}^{n}} k_{\lambda}\left(\|x\|_{\alpha},\|y\|_{\alpha}\right) f(x) g(y) d x d y \\
<\frac{\Gamma^{n}\left(\frac{1}{\alpha}\right)}{\alpha^{n-1} \Gamma\left(\frac{n}{\alpha}\right)} k_{\lambda}(r)\left[\int_{\mathbf{R}_{+}^{n}}\|x\|_{\alpha}^{p\left(n-\frac{\lambda}{r}\right)-n} f^{p}(x) d x\right]^{\frac{1}{p}} \\
\quad \times\left[\int_{\mathbf{R}_{+}^{n}}\|y\|_{\alpha}^{q\left(n-\frac{\lambda}{s}\right)-n} g^{q}(y) d y\right]^{\frac{1}{q}}
\end{gathered}
$$

where the constant factor $\frac{\Gamma^{n}\left(\frac{1}{\alpha}\right)}{\alpha^{n-1} \Gamma\left(\frac{n}{\alpha}\right)} k_{\lambda}(r)$ is the best possible.

Also, the equivalent form of (110), the reverses, the Hardy-type inequalities, the operator expressions, and many particular examples are provided. Some other results of multidimensional Hilbert-type integral inequalities are discussed by [151, 152].

For $n=2$ in (109), or $\alpha=n=1$ in (110), we reduce the following Yang-Hilbert-type integral inequality:

$$
\begin{aligned}
& \int_{0}^{\infty} \int_{0}^{\infty} k_{\lambda}(x, y) f(x) g(y) d x d y \\
& \quad<k_{\lambda}(r)\left[\int_{0}^{\infty} x^{p\left(1-\frac{\lambda}{r}\right)-1} f^{p}(x) d x\right]^{\frac{1}{p}}\left[\int_{0}^{\infty} y^{q\left(1-\frac{\lambda}{s}\right)-1} g^{q}(y) d y\right]^{\frac{1}{q}},
\end{aligned}
$$

where the constant factor $k_{\lambda}(r)$ is the best possible. The equivalent form of (111) is obtained as follows (cf. [147], Theorem 2.2.1):

$$
\int_{0}^{\infty} y^{\frac{p \lambda}{s}-1}\left(\int_{0}^{\infty} k_{\lambda}(x, y) f(x) d x\right)^{p} d y<k_{\lambda}^{p}(r) \int_{0}^{\infty} x^{p\left(1-\frac{\lambda}{r}\right)-1} f^{p}(x) d x
$$

where the constant factor $k_{\lambda}^{p}(r)$ is the best possible. 
For $\lambda=1, r=q, s=p,(111)$ and (112) reduce, respectively, to (33) and (34). Hence, YangHilbert-type integral inequalities are extensions of Hardy-Hilbert-type integral inequalities.

If we replace $y$ and $g\left(\frac{1}{y}\right)$ to $\frac{1}{y}$ and $y^{2-\lambda} g(y)$ in (111) and (112), then we obtain the following equivalent inequalities with the non-homogeneous kernel and the best possible constant factors $(c f .[153])$ :

$$
\begin{aligned}
& \int_{0}^{\infty} \int_{0}^{\infty} k_{\lambda}(x y, 1) f(x) g(y) d x d y \\
& <k_{\lambda}(r)\left[\int_{0}^{\infty} x^{p\left(1-\frac{\lambda}{r}\right)-1} f^{p}(x) d x\right]^{\frac{1}{p}}\left[\int_{0}^{\infty} y^{q\left(1-\frac{\lambda}{r}\right)-1} g^{q}(y) d y\right]^{\frac{1}{q}}, \\
& \int_{0}^{\infty} y^{\frac{p \lambda}{r}-1}\left(\int_{0}^{\infty} k_{\lambda}(x y, 1) f(x) d x\right)^{p} d z<k_{\lambda}^{p}(r) \int_{0}^{\infty} x^{p\left(1-\frac{\lambda}{r}\right)-1} f^{p}(x) d x .
\end{aligned}
$$

Replacing $x$ and $f\left(\frac{1}{x}\right)$ to $\frac{1}{x}$ and $x^{2-\lambda} f(x)$ in (111) and (112), we also obtain the following equivalent inequalities with the non-homogeneous kernel and the best possible constant factors:

$$
\begin{aligned}
& \int_{0}^{\infty} \int_{0}^{\infty} k_{\lambda}(1, x y) f(x) g(y) d x d y \\
& <k_{\lambda}(r)\left[\int_{0}^{\infty} x^{p\left(1-\frac{\lambda}{s}\right)-1} f^{p}(x) d x\right]^{\frac{1}{p}}\left[\int_{0}^{\infty} y^{q\left(1-\frac{\lambda}{s}\right)-1} g^{q}(y) d y\right]^{\frac{1}{q}}, \\
& \int_{0}^{\infty} y^{\frac{p \lambda}{s}-1}\left(\int_{0}^{\infty} k_{\lambda}(1, x y) f(x) d x\right)^{p} d y<k_{\lambda}^{p}(r) \int_{0}^{\infty} x^{p\left(1-\frac{\lambda}{s}\right)-1} f^{p}(x) d x .
\end{aligned}
$$

It is evident that (111)-(116) are equivalent. In particular, if $k_{\lambda}(x, y)$ is symmetric, then we have

$$
\begin{aligned}
& \int_{0}^{\infty} \int_{0}^{\infty} k_{\lambda}(x y, 1) f(x) g(y) d x d y \\
& \quad<k_{\lambda}(r) \min _{a \in\{r, s\}}\left\{\left[\int_{0}^{\infty} x^{p\left(1-\frac{\lambda}{a}\right)-1} f^{p}(x) d x\right]^{\frac{1}{p}}\left[\int_{0}^{\infty} y^{q\left(1-\frac{\lambda}{a}\right)-1} g^{q}(y) d y\right]^{\frac{1}{q}}\right\} .
\end{aligned}
$$

The above inequalities are some refinements of (33)-(36).

\subsection{Discrete Yang-Hilbert-type inequalities}

In 2011, Yang [148] (Theorem 4.2.3) gave an extension of (35) and (36) as follows.

If $p>1, \frac{1}{p}+\frac{1}{q}=1, \lambda_{1}, \lambda_{2} \in \mathbf{R}, \lambda_{1}+\lambda_{2}=\lambda, k_{\lambda}(x, y)(\geq 0)$ is a finite homogeneous function of degree $-\lambda$ in $\mathbf{R}_{+}^{2}$,

$$
k\left(\lambda_{1}\right)=\int_{0}^{\infty} k_{\lambda}(u, 1) u^{\lambda_{1}-1} d u \in \mathbf{R}_{+}
$$

$k_{\lambda}(x, y) \frac{1}{x^{1-\lambda_{2}}}\left(k_{\lambda}(x, y) \frac{1}{y^{1-\lambda_{1}}}\right)$ is decreasing with respect to $x(y)>0, a_{m}, b_{n} \geq 0,0<$ $\sum_{m=1}^{\infty} m^{p\left(1-\lambda_{1}\right)-1} a_{m}^{p}<\infty, 0<\sum_{n=1}^{\infty} n^{q\left(1-\lambda_{2}\right)-1} b_{n}^{q}<\infty$, then we have the following equivalent 
discrete Yang-Hilbert-type inequalities:

$$
\begin{aligned}
& \sum_{n=1}^{\infty} \sum_{m=1}^{\infty} k_{\lambda}(m, n) a_{m} b_{n} \\
& <k\left(\lambda_{1}\right)\left[\sum_{m=1}^{\infty} m^{p\left(1-\lambda_{1}\right)-1} a_{m}^{p}\right]^{\frac{1}{p}}\left[\sum_{n=1}^{\infty} n^{q\left(1-\lambda_{2}\right)-1} b_{n}^{q}\right]^{\frac{1}{q}}, \\
& \sum_{n=1}^{\infty} n^{p \lambda_{2}-1}\left(\sum_{m=1}^{\infty} k_{\lambda}(m, n) a_{m}\right)^{p}<\left(k\left(\lambda_{1}\right)\right)^{p} \sum_{m=1}^{\infty} m^{p\left(1-\lambda_{1}\right)-1} a_{m}^{p},
\end{aligned}
$$

where the constant factors $k\left(\lambda_{1}\right)$ and $\left(k\left(\lambda_{1}\right)\right)^{p}$ are the best possible.

In this reference, some extensions of (118) and (119), the reverses, the operator expressions, and some particular examples are provided.

The following multiple inequalities are considered (cf. [148], Corollary 6.1.7).

If $n \in \mathbf{N} \backslash\{1\}, p_{i}>1, \lambda_{i} \in \mathbf{R}(i=1,2, \ldots, n), \sum_{i=1}^{n} \frac{1}{p_{i}}=1, \sum_{i=1}^{n} \lambda_{i}=\lambda, \frac{1}{q_{n}}=1-\frac{1}{p_{n}}, k_{\lambda}\left(x_{1}, \ldots, x_{n}\right)$ $(\geq 0)$ is a finite homogeneous function of degree $-\lambda$ in $\mathbf{R}_{+}^{n}, k_{\lambda}\left(x_{1}, \ldots, x_{n}\right) \frac{1}{x_{i}^{1-\lambda_{i}}}$ is decreasing with respect to $x_{i}>0(i=1, \ldots, n)$,

$$
k\left(\lambda_{1}, \ldots, \lambda_{n-1}\right)=\int_{0}^{\infty} \cdots \int_{0}^{\infty} k_{\lambda}\left(u_{1}, \ldots, u_{n-1}, 1\right) \prod_{j=1}^{n-1} u_{j}^{\lambda_{j}-1} d u_{1} \cdots d u_{n-1} \in \mathbf{R}_{+}
$$

$a_{m_{i}}^{(i)} \geq 0$ and $0<\sum_{m_{i}=1}^{\infty} m_{i}^{p_{i}\left(1-\lambda_{i}\right)-1}\left(a_{m_{i}}^{(i)}\right)^{p_{i}}<\infty(i=1,2, \ldots, n)$, then we still have the following multiple equivalent inequalities:

$$
\begin{aligned}
& \sum_{m_{n}=1}^{\infty} \cdots \sum_{m_{1}=1}^{\infty} k_{\lambda}\left(m_{1}, \ldots, m_{n}\right) \prod_{i=1}^{n} a_{m_{i}}^{(i)} \\
& \quad<k\left(\lambda_{1}, \ldots, \lambda_{n-1}\right) \prod_{i=1}^{n}\left[\sum_{m_{i}=1}^{\infty} m_{i}^{p_{i}\left(1-\lambda_{i}\right)-1}\left(a_{m_{i}}^{(i)}\right)^{p_{i}}\right]^{\frac{1}{p_{i}}}, \\
& \sum_{m_{n}=1}^{\infty} m_{n}^{q_{n} \lambda_{n}-1}\left[\sum_{m_{n-1}=1}^{\infty} \cdots \sum_{m_{1}=1}^{\infty} k_{\lambda}\left(m_{1}, \ldots, m_{n}\right) \prod_{i=1}^{n-1} a_{m_{i}}^{(i)}\right]^{q_{n}} \\
& \quad<\left(k\left(\lambda_{1}, \ldots, \lambda_{n-1}\right)\right)^{q_{n}} \prod_{i=1}^{n-1}\left[\sum_{m_{i}=1}^{\infty} m_{i}^{p_{i}\left(1-\lambda_{i}\right)-1}\left(a_{m_{i}}^{(i)}\right)^{p_{i}}\right]^{\frac{q_{n}}{p_{i}}},
\end{aligned}
$$

where the constant factors $k\left(\lambda_{1}, \ldots, \lambda_{n-1}\right)$ and $\left(k\left(\lambda_{1}, \ldots, \lambda_{n-1}\right)\right)^{q_{n}}$ are the best possible.

In this book, the reverses of (120) and (121) are also considered. For $n=2,(120)$ and (121) reduce, respectively, to (120) and (121); for $\lambda=1, \lambda_{i}=1-\frac{1}{p_{i}}(i=1, \ldots, n),(120)$ reduces to (54).

In 2014, Yang [154] (Corollary 3.2) gave the following results.

Suppose that $i_{0}, j_{0} \in \mathbf{N}, \alpha, \beta>0, \lambda_{1}<i_{0}, \lambda_{2}<j_{0}, \lambda_{1}+\lambda=\lambda, k_{\lambda}(x, y)(>0)$ is a finite homogeneous function of degree $-\lambda$ in $\mathbf{R}_{+}^{2}$, which is decreasing with respect to $x(y)>0$, there exists a constant $0<\delta_{0}<j_{0}-\lambda_{2}$, such that for any $\tilde{\lambda}_{1} \in\left(\lambda_{1}-\delta_{0}, \lambda_{1}+\delta_{0}\right), k\left(\tilde{\lambda}_{1}\right)=$ $\int_{0}^{\infty} k_{\lambda}(u, 1) u^{\tilde{\lambda}_{1}-1} d u \in \mathbf{R}_{+}$, and there exists a constant $\delta_{1}<\lambda_{1}-\delta_{0}$, satisfying $k_{\lambda}(u, 1) \leq \frac{L}{u^{\delta_{1}}}$ 
$(u \in(0, \infty))$. If $p>1, \frac{1}{p}+\frac{1}{q}=1, a(m), b(n) \geq 0,0<\sum_{m \in \mathbf{N}^{i_{0}}}\|m\|_{\alpha}^{p\left(i_{0}-\lambda_{1}\right)-i_{0}} a^{p}(m)<\infty$, $0<\sum_{n \in \mathrm{N}^{j_{0}}}\|n\|_{\beta}^{q\left(j_{0}-\lambda_{2}\right)-j_{0}} b^{q}(n)<\infty$,

$$
K\left(\lambda_{1}\right)=\left(\frac{\Gamma^{i_{0}}\left(\frac{1}{\alpha}\right)}{\alpha^{i_{0}-1} \Gamma\left(\frac{i_{0}}{\alpha}\right)}\right)^{\frac{1}{q}}\left(\frac{\Gamma^{j_{0}}\left(\frac{1}{\beta}\right)}{\beta^{j_{0}-1} \Gamma\left(\frac{j_{0}}{\beta}\right)}\right) k\left(\lambda_{1}\right),
$$

then we have the following inequality:

$$
\begin{aligned}
& \sum_{n \in \mathbf{N}^{j} 0} \sum_{m \in \mathbf{N}^{i_{0}}} k_{\lambda}\left(\|m\|_{\alpha},\|n\|_{\beta}\right) a(m) b(n) \\
& <K\left(\lambda_{1}\right)\left[\sum_{m \in \mathbf{N}^{i_{0}}}\|m\|_{\alpha}^{p\left(i_{0}-\lambda_{1}\right)-i_{0}} a^{p}(m)\right]^{\frac{1}{p}}\left[\sum_{n \in \mathbf{N}^{j}}\|n\|_{\beta}^{q\left(j_{0}-\lambda_{2}\right)-j_{0}} b^{q}(n)\right]^{\frac{1}{q}}, \\
& \sum_{n \in \mathbf{N}^{j} 0}\|n\|_{\beta}^{p \lambda_{2}-j_{0}}\left(\sum_{m \in \mathbf{N}^{i_{0}}} k_{\lambda}\left(\|m\|_{\alpha},\|n\|_{\beta}\right) a(m)\right)^{p} \\
& <K^{p}\left(\lambda_{1}\right) \sum_{m \in \mathbf{N}^{i_{0}}}\|m\|_{\alpha}^{p\left(i_{0}-\lambda_{1}\right)-i_{0}} a^{p}(m),
\end{aligned}
$$

where the constant factors $K\left(\lambda_{1}\right)$ and $K^{p}\left(\lambda_{1}\right)$ are the best possible.

For $i_{0}=j_{0}=\alpha=\beta=1,(122)$ and (123) also reduce, respectively, to (118) and (119). In this chapter, the reverses and the operator expressions of (122) and (123) are provided. A composition formula of the operators is developed.

\subsection{Half-discrete Yang-Hilbert-type inequalities}

In 2014, Yang and Debnath [155] (Theorem 6.1) gave the following results.

Suppose that $m \in \mathbf{N}, p_{i}>1, \lambda_{i} \in \mathbf{R}(i=1,2, \ldots, m+1), \sum_{i=1}^{m+1} \frac{1}{p_{i}}=1, \sum_{i=1}^{m+1} \lambda_{i}=\lambda, \frac{1}{p}=1-$ $\frac{1}{p_{m+1}}, k_{\lambda}\left(x_{1}, \ldots, x_{n}\right)(\geq 0)$ is a finite homogeneous function of degree $-\lambda$ in $\mathbf{R}_{+}^{n}$, there exists a constant $\delta_{0}>0$, such that for any $\tilde{\lambda}_{i} \in\left(\lambda_{i}-\delta_{0}, \lambda_{i}+\delta_{0}\right), \sum_{i=1}^{m+1} \tilde{\lambda}_{i}=\lambda, k_{\lambda}\left(x_{1}, \ldots, x_{m}, y\right) \frac{1}{y^{1-\tilde{\lambda}_{m+1}}}$ is strictly decreasing with respect to $y>0$, and

$$
k\left(\tilde{\lambda}_{m+1}\right)=\int_{0}^{\infty} \cdots \int_{0}^{\infty} k_{\lambda}\left(u_{1}, \ldots, u_{m}, 1\right) \prod_{j=1}^{m} u_{j}^{\tilde{\lambda}_{j}-1} d u_{1} \cdots d u_{m} \in \mathbf{R}_{+} .
$$

If $f_{i}\left(x_{i}\right), a_{n} \geq 0$ and $0<\sum_{n=1}^{\infty} n^{p_{m+1}\left(1-\lambda_{m+1}\right)-1} a_{n}^{p_{m+1}}<\infty$,

$$
0<\int_{0}^{\infty} t^{p_{i}\left(1-\lambda_{i}\right)-1} f_{i}^{p_{i}}(t) d t<\infty \quad(i=1, \ldots, m)
$$

then we still have the following equivalent inequalities:

$$
\begin{aligned}
& \sum_{n=1}^{\infty} \int_{0}^{\infty} \ldots \int_{0}^{\infty} k_{\lambda}\left(x_{1}, \ldots, x_{m}, n\right) a_{n} \prod_{i=1}^{m} f_{i}\left(x_{i}\right) d x_{1} \cdots d x_{m} \\
& \quad<k\left(\lambda_{m+1}\right)\left[\sum_{n=1}^{\infty} n^{p_{m+1}\left(1-\lambda_{m+1}\right)-1} a_{n}^{p_{m+1}}\right]^{\frac{1}{p_{m+1}}} \prod_{i=1}^{m}\left[\int_{0}^{\infty} t^{p_{i}\left(1-\lambda_{i}\right)-1} f_{i}^{p_{i}}(t) d t\right]^{\frac{1}{p_{i}}},
\end{aligned}
$$




$$
\begin{aligned}
& \sum_{n=1}^{\infty} n^{p \lambda_{m+1}-1}\left(\int_{0}^{\infty} \cdots \int_{0}^{\infty} k_{\lambda}\left(x_{1}, \ldots, x_{m}, n\right) \prod_{i=1}^{m} f_{i}\left(x_{i}\right) d x_{1} \cdots d x_{m}\right)^{p} \\
& \quad<k^{p}\left(\lambda_{m+1}\right) \prod_{i=1}^{m}\left[\int_{0}^{\infty} t^{p_{i}\left(1-\lambda_{i}\right)-1} f_{i}^{p_{i}}(t) d t\right]^{\frac{p}{p_{i}}},
\end{aligned}
$$

where the constant factors

$$
k\left(\lambda_{m+1}\right)=\int_{0}^{\infty} \cdots \int_{0}^{\infty} k_{\lambda}\left(u_{1}, \ldots, u_{m}, 1\right) \prod_{j=1}^{m} u_{j}^{\lambda_{j}-1} d u_{1} \cdots d u_{m}
$$

and $k^{p}\left(\lambda_{m+1}\right)$ are the best possible.

In this book, the reverses, the operator expressions, and some particular examples are provided. Some other kinds of multiple half-discrete Hilbert-type inequalities are discussed in $[149,150]$. The composition formula of operators are given by [156].

In Corollary 5.3 of this book, Yang also gave the following multi-dimensional halfdiscrete inequalities.

Suppose that $m, s \in \mathbf{N}, \alpha, \beta>0, \lambda_{1}, \lambda_{2} \in \mathbf{R}, \lambda_{1}+\lambda_{2}=\lambda, k_{\lambda}(x, y)(\geq 0)$ is a finite homogeneous function of degree $-\lambda$ in $\mathbf{R}_{+}^{2}, k_{\lambda}(x, y) \frac{1}{y^{s-\lambda_{2}}}$ is decreasing with respect to $y>0$, and strictly decreasing in an interval $I \subset(1, \infty), k\left(\lambda_{1}\right)=\int_{0}^{\infty} k_{\lambda}(u, 1) u^{\lambda_{1}-1} d u \in \mathbf{R}_{+}$,

$$
K\left(\lambda_{1}\right)=\left(\frac{\Gamma^{m}\left(\frac{1}{\alpha}\right)}{\alpha^{m-1} \Gamma\left(\frac{m}{\alpha}\right)}\right)^{\frac{1}{q}}\left(\frac{\Gamma^{s}\left(\frac{1}{\beta}\right)}{\beta^{s-1} \Gamma\left(\frac{s}{\beta}\right)}\right) k\left(\lambda_{1}\right) .
$$

If $p>1, \frac{1}{p}+\frac{1}{q}=1, f(x), a(n) \geq 0,0<\int_{\mathbf{R}_{+}^{m}}\|x\|_{\alpha}^{p\left(m-\lambda_{1}\right)-m} f^{p}(x) d x<\infty$, and $0<$ $\sum_{n \in \mathbf{N}^{s}}\|n\|_{\beta}^{q\left(s-\lambda_{2}\right)-s} a^{q}(n)<\infty$, then we have the following equivalent inequalities:

$$
\begin{aligned}
& \int_{\mathbf{R}_{+}^{m}} \sum_{n \in \mathbf{N}^{s}} k_{\lambda}\left(\|x\|_{\alpha},\|n\|_{\beta}\right) f(x) a(n) d x \\
& <K\left(\lambda_{1}\right)\left[\int_{\mathbf{R}_{+}^{m}}\|x\|_{\alpha}^{p\left(m-\lambda_{1}\right)-m} f^{p}(x) d x\right]^{\frac{1}{p}}\left[\sum_{n \in \mathbf{N}^{s}}\|n\|_{\beta}^{q\left(s-\lambda_{2}\right)-s} a^{q}(n)\right]^{\frac{1}{q}}, \\
& \sum_{n \in \mathbf{N}^{s}}\|n\|_{\beta}^{p \lambda_{2}-s}\left(\int_{\mathbf{R}_{+}^{m}} k_{\lambda}\left(\|x\|_{\alpha},\|n\|_{\beta}\right) f(x) d x\right)^{p} \\
& <\left(K\left(\lambda_{1}\right)\right)^{p} \int_{\mathbf{R}_{+}^{m}}\|x\|_{\alpha}^{p\left(m-\lambda_{1}\right)-m} f^{p}(x) d x, \\
& \int_{\mathbf{R}_{+}^{m}}\|x\|_{\alpha}^{q \lambda_{1}-m}\left(\sum_{n \in \mathbf{N}^{s}} k_{\lambda}\left(\|x\|_{\alpha},\|n\|_{\beta}\right) a(n)\right)^{q} d x<\left(K\left(\lambda_{1}\right)\right)^{q} \sum_{n \in \mathbf{N}^{s}}\|n\|_{\beta}^{q\left(s-\lambda_{2}\right)-s} a^{q}(n),
\end{aligned}
$$

where the constant factors $K\left(\lambda_{1}\right),\left(K\left(\lambda_{1}\right)\right)^{p}$, and $\left(K\left(\lambda_{1}\right)\right)^{q}$ are the best possible.

For $m=s=\alpha=\beta=1$, (126), (127), and (128) reduce to the following equivalent halfdiscrete Yang-Hilbert-type inequalities with the best possible constant factors:

$$
\begin{aligned}
& \int_{0}^{\infty} \sum_{n=1}^{\infty} k_{\lambda}(x, n) f(x) a_{n} d x \\
& \quad<k\left(\lambda_{1}\right)\left[\int_{0}^{\infty} x^{p\left(1-\lambda_{1}\right)-1} f^{p}(x) d x\right]^{\frac{1}{p}}\left[\sum_{n=1}^{\infty} n^{q\left(1-\lambda_{2}\right)-1} a_{n}^{q}\right]^{\frac{1}{q}},
\end{aligned}
$$




$$
\begin{aligned}
& \sum_{n=1}^{\infty} n^{p \lambda_{2}-1}\left(\int_{0}^{\infty} k_{\lambda}(x, n) f(x) d x\right)^{p}<\left(k\left(\lambda_{1}\right)\right)^{p} \int_{0}^{\infty} x^{p\left(1-\lambda_{1}\right)-1} f^{p}(x) d x, \\
& \int_{0}^{\infty} x^{q \lambda_{1}-1}\left(\sum_{n=1}^{\infty} k_{\lambda}(x, n) a_{n}\right)^{q} d x<\left(k\left(\lambda_{1}\right)\right)^{q} \sum_{n=1}^{\infty} n^{q\left(1-\lambda_{2}\right)-1} a_{n}^{q} .
\end{aligned}
$$

Also, for $m=1,(124)$ and (125) reduce, respectively, to (129) and (130).

Replacing $x$ to $\frac{1}{x}, x^{\lambda-2} f\left(\frac{1}{x}\right)$ to $f(x)$ in (129), (130), and (131), we have the following equivalent inequalities with the non-homogeneous kernel and the best possible constant factors:

$$
\begin{aligned}
& \int_{0}^{\infty} \sum_{n=1}^{\infty} k_{\lambda}(1, x n) f(x) a_{n} d x \\
& <k\left(\lambda_{1}\right)\left[\int_{0}^{\infty} x^{p\left(1-\lambda_{2}\right)-1} f^{p}(x) d x\right]^{\frac{1}{p}}\left[\sum_{n=1}^{\infty} n^{q\left(1-\lambda_{2}\right)-1} a_{n}^{q}\right]^{\frac{1}{q}}, \\
& \sum_{n=1}^{\infty} n^{p \lambda_{2}-1}\left(\int_{0}^{\infty} k_{\lambda}(1, x n) f(x) d x\right)^{p}<\left(k\left(\lambda_{1}\right)\right)^{p} \int_{0}^{\infty} x^{p\left(1-\lambda_{2}\right)-1} f^{p}(x) d x, \\
& \int_{0}^{\infty} x^{q \lambda_{2}-1}\left(\sum_{n=1}^{\infty} k_{\lambda}(1, x n) a_{n}\right)^{q} d x<\left(k\left(\lambda_{1}\right)\right)^{q} \sum_{n=1}^{\infty} n^{q\left(1-\lambda_{2}\right)-1} a_{n}^{q} .
\end{aligned}
$$

The above half-discrete inequalities are some refinements of (37)-(40).

\subsection{Some simple Hilbert-type inequalities}

If the Hilbert-type inequality relates to a simple symmetric homogeneous kernel of degree -1 and the best constant factor is a more brief form, which does not relate to any conjugate exponents (such as (2)), then we call it simple Hilbert-type integral inequality. Its series analog (if it exists) is called a simple Hilbert-type inequality. If the simple homogeneous kernel is of $-\lambda$ degree $(\lambda>0)$ with a parameter $\lambda$ and the inequality cannot be obtained by a simple transform to a simple Hilbert-type integral inequality, then we call it a simple Hilbert-type integral inequality with a parameter.

For example, we call the following integral inequality (i.e. (2)):

$$
\int_{0}^{\infty} \int_{0}^{\infty} \frac{f(x) g(y)}{x+y} d x d y<\pi\left(\int_{0}^{\infty} f^{2}(x) d x \int_{0}^{\infty} g^{2}(y) d y\right)^{\frac{1}{2}}
$$

and the following H-L-P inequalities (for $p=2$ in (37) and (41)):

$$
\begin{aligned}
& \int_{0}^{\infty} \int_{0}^{\infty} \frac{f(x) g(y)}{\max \{x, y\}} d x d y<4\left(\int_{0}^{\infty} f^{2}(x) d x \int_{0}^{\infty} g^{2}(y) d y\right)^{\frac{1}{2}} \\
& \int_{0}^{\infty} \int_{0}^{\infty} \frac{\ln \left(\frac{x}{y}\right) f(x) g(y)}{x-y} d x d y<\pi^{2}\left(\int_{0}^{\infty} f^{2}(x) d x \int_{0}^{\infty} g^{2}(y) d y\right)^{\frac{1}{2}}
\end{aligned}
$$

simple Hilbert-type integral inequalities.

In 2006, Yang [21] gave the following simple Hilbert-type integral inequality:

$$
\int_{0}^{\infty} \int_{0}^{\infty} \frac{\left|\ln \left(\frac{x}{y}\right)\right| f(x) g(y)}{\max \{x, y\}} d x d y<8\left(\int_{0}^{\infty} f^{2}(x) d x \int_{0}^{\infty} g^{2}(y) d y\right)^{\frac{1}{2}}
$$


In 2008, Yang [23] and [157] gave the following simple Hilbert-type integral inequalities:

$$
\begin{aligned}
& \int_{0}^{\infty} \int_{0}^{\infty} \frac{\left|\ln \left(\frac{x}{y}\right)\right| f(x) g(y)}{x+y} d x d y<c_{0}\left(\int_{0}^{\infty} f^{2}(x) d x \int_{0}^{\infty} g^{2}(y) d y\right)^{\frac{1}{2}} \\
& \int_{0}^{\infty} \int_{0}^{\infty} \frac{\arctan \sqrt{\frac{x}{y}}}{x+y} f(x) g(y) d x d y<\frac{\pi^{2}}{4}\left(\int_{0}^{\infty} f^{2}(x) d x \int_{0}^{\infty} g^{2}(y) d y\right)^{\frac{1}{2}}
\end{aligned}
$$

where $c_{0}=8 \sum_{n=1}^{\infty} \frac{(-1)^{n}}{(2 n-1)^{2}}=7.3277^{+}$.

In 2005 , Yang $[158,159]$ gave a simple Hilbert-type integral inequality with a parameter $\lambda \in(0,1):$

$$
\begin{aligned}
& \int_{0}^{\infty} \int_{0}^{\infty} \frac{f(x) g(y)}{|x-y|^{\lambda}} d x d y \\
& \quad<2 B\left(1-\lambda, \frac{\lambda}{2}\right)\left(\int_{0}^{\infty} x^{1-\lambda} f^{2}(x) d x \int_{0}^{\infty} y^{1-\lambda} g^{2}(y) d y\right)^{\frac{1}{2}}
\end{aligned}
$$

As in (16),

$$
\begin{aligned}
& \int_{0}^{\infty} \int_{0}^{\infty} \frac{f(x) g(y)}{(x+y)^{\lambda}} d x d y \\
& \quad<B\left(\frac{\lambda}{2}, \frac{\lambda}{2}\right)\left(\int_{0}^{\infty} x^{1-\lambda} f^{2}(x) d x \int_{0}^{\infty} y^{1-\lambda} g^{2}(y) d y\right)^{\frac{1}{2}}
\end{aligned}
$$

is called a simple Hilbert-type integral inequality with the parameter $\lambda \in(0, \infty)$.

Also we find the following simple Hilbert-type inequality:

$$
\begin{aligned}
& \sum_{n=1}^{\infty} \sum_{m=1}^{\infty} \frac{a_{m} b_{n}}{m+n}<\pi\left(\sum_{m=1}^{\infty} a_{m}^{2} \sum_{n=1}^{\infty} b_{n}^{2}\right)^{\frac{1}{2}}, \\
& \sum_{n=1}^{\infty} \sum_{m=1}^{\infty} \frac{a_{m} b_{n}}{\max \{m, n\}}<4\left(\sum_{m=1}^{\infty} a_{m}^{2} \sum_{n=1}^{\infty} b_{n}^{2}\right)^{\frac{1}{2}}, \\
& \sum_{n=1}^{\infty} \sum_{m=1}^{\infty} \frac{\ln \left(\frac{m}{n}\right) a_{m} b_{n}}{m-n}<\pi^{2}\left(\sum_{m=1}^{\infty} a_{m}^{2} \sum_{n=1}^{\infty} b_{n}^{2}\right)^{\frac{1}{2}} .
\end{aligned}
$$

In (47), for $p=q=2$, we have

$$
\sum_{n=1}^{\infty} \sum_{m=1}^{\infty} \frac{\left|\ln \left(\frac{m}{n}\right)\right| a_{m} b_{n}}{\max \{m, n\}}<8\left(\sum_{m=1}^{\infty} a_{m}^{2} \sum_{n=1}^{\infty} b_{n}^{2}\right)^{\frac{1}{2}}
$$

In 2010, Xin and Yang [24] gave

$$
\sum_{n=1}^{\infty} \sum_{m=1}^{\infty} \frac{\left|\ln \left(\frac{m}{n}\right)\right| a_{m} b_{n}}{m+n}<c_{0}\left(\sum_{m=1}^{\infty} a_{m}^{2} \sum_{n=1}^{\infty} b_{n}^{2}\right)^{\frac{1}{2}}
$$


where $c_{0}=8 \sum_{n=1}^{\infty} \frac{(-1)^{n}}{(2 n-1)^{2}}=7.3277^{+}$. Inequalities (143) and (144) are new simple Hilberttype inequalities. We still have a simple Hilbert-type inequality with a parameter $\lambda \in(0,4]$ as follows (cf. [51]):

$$
\sum_{n=1}^{\infty} \sum_{m=1}^{\infty} \frac{a_{m} b_{n}}{(m+n)^{\lambda}}<B\left(\frac{\lambda}{2}, \frac{\lambda}{2}\right)\left(\sum_{m=1}^{\infty} m^{1-\lambda} a_{m}^{2} \sum_{n=1}^{\infty} n^{1-\lambda} b_{n}^{2}\right)^{\frac{1}{2}} .
$$

Some simple half-discrete Hilbert-type inequalities are also listed as follows:

$$
\begin{aligned}
& \int_{0}^{\infty} \sum_{n=1}^{\infty} \frac{a_{n}}{x+n} f(x) d x<\pi\left(\int_{0}^{\infty} f^{2}(x) d x \sum_{n=1}^{\infty} a_{n}^{2}\right)^{\frac{1}{2}}, \\
& \int_{0}^{\infty} \sum_{n=1}^{\infty} \frac{a_{n}}{\max \{x, n\}} f(x) d x<4\left(\int_{0}^{\infty} f^{2}(x) d x \sum_{n=1}^{\infty} a_{n}^{2}\right)^{\frac{1}{2}}, \\
& \int_{0}^{\infty} \sum_{n=1}^{\infty} \frac{\ln \left(\frac{x}{n}\right) a_{n}}{x-n} f(x) d x<\pi^{2}\left(\int_{0}^{\infty} f^{2}(x) d x \sum_{n=1}^{\infty} a_{n}^{2}\right)^{\frac{1}{2}}, \\
& \int_{0}^{\infty} \sum_{n=1}^{\infty} \frac{\left|\ln \left(\frac{x}{n}\right)\right| a_{n}}{\max \{x, n\}} f(x) d x<8\left(\int_{0}^{\infty} f^{2}(x) d x \sum_{n=1}^{\infty} a_{n}^{2}\right)^{\frac{1}{2}}, \\
& \int_{0}^{\infty} \sum_{n=1}^{\infty} \frac{\left|\ln \left(\frac{x}{n}\right)\right| a_{n}}{x+n} f(x) d x<c_{0}\left(\int_{0}^{\infty} f^{2}(x) d x \sum_{n=1}^{\infty} a_{n}^{2}\right)^{\frac{1}{2}} .
\end{aligned}
$$

\section{Competing interests}

The authors declare that they have no competing interests.

\section{Authors' contributions}

BY carried out the mathematical studies, participated in the sequence alignment and drafted the manuscript. QC participated in the design of the study and performed the numerical analysis. All authors read and approved the final manuscript.

\section{Author details}

'Department of Computer Science, Guangdong University of Education, Guangzhou, Guangdong 510303, P.R. China.

${ }^{2}$ Department of Mathematics, Guangdong University of Education, Guangzhou, Guangdong 510303, P.R. China.

\section{Acknowledgements}

The authors wish to express their thanks to the referees for their careful reading of the manuscript and for their valuable suggestions. This work is supported by the National Natural Science Foundation (No. 61370186), and the Science and Technology Planning Project of Guangzhou (No. 2014J4100032, No. 201510010203). We are grateful for their help.

Received: 24 March 2015 Accepted: 16 September 2015 Published online: 26 September 2015

\section{References}

1. Weyl, H: Singulare integral Gleichungen mit besonderer Berücksichtigung des Fourierschen integral theorems. Inaugeral-Dissertation, Gottingen (1908)

2. Schur, I: Bemerkungen für Theorie der beschränkten Bilinearformen mit unendlich vielen Veränderlichen. J. Math. $140,1-28(1911)$

3. Hardy, GH, Littlewood, JE, Pólya, G: Inequalities. Cambridge University Press, Cambridge (1934)

4. Mitrinović, DS, Pečarić, JE, Fink, AM: Inequalities Involving Functions and Their Integrals and Derivatives. Kluwer Academic, Boston (1991)

5. Kuang, J: Applied Inequalities. Shandong Science Technic Press, Jinan (2004)

6. Hu, K: Some Problems in Analysis Inequalities. Wuhan University Press, Wuhan (2007)

7. Wilhelm, M: On the spectrum of Hilbert's matrix. Am. J. Math. 72, 699-704 (1950)

8. Carleman, T: Sur les Équations Integrals Singulières a Noyau Reel et Symetrique. Almqvist \& Wiksell, Uppsala (1923)

9. Ingham, AE: A note on Hilbert's inequality. J. Lond. Math. Soc. 11, 237-240 (1936)

10. Yang, B: On a new Hardy-Hilbert's type inequality. Math. Inequal. Appl. 7(3), 355-363 (2004)

11. Yang, B: A more accurate Hardy-Hilbert's type inequality. J. Xinyang Norm. Univ. 18(2), 140-142 (2005) 
12. Yang, B: A more accurate Hilbert-type inequality. Coll. Math. 21(5), 99-102 (2005)

13. Yang, B: On a more accurate Hardy-Hilbert's type inequality and its applications. Acta Math. Sin. 49(3), 363-368 (2006)

14. Yang, B: A more accurate Hilbert type inequality. J. Math. 27(6), 673-678 (2007)

15. Yang, B: On an extension of Hardy-Hilbert's type inequality and a reverse. Acta Math. Sin. Chin. Ser. 50(4), 861-868 (2007)

16. Yang, B: On a more accurate Hilbert's type inequality. Int. Math. Forum 2(37), 1831-1837 (2007)

17. Zhong, J, Yang, B: On an extension of a more accurate Hilbert-type inequality. J. Zhejiang Univ. Sci. Ed. 35(2), 121-124 (2008)

18. Hardy, GH: Note on a theorem of Hilbert concerning series of positive term. Proc. Lond. Math. Soc. 23, 45-46 (1925)

19. Levin, V: Two remarks on Hilbert's double series theorem. J. Indian Math. Soc. 11, 111-115 (1937)

20. Bonsall, FF: Inequalities with non-conjugate parameter. Q. J. Math. 2(1), 135-150 (1951)

21. Yang, B: On a basic Hilbert-type inequality. J. Guangdong Educ. Inst. 26(3), 1-5 (2006)

22. Yang, B: A Hilbert-type inequality with two pairs of conjugate exponents. J. Jilin Univ. Sci. Ed. 45(4), 524-528 (2007)

23. Yang, B: On a basic Hilbert-type integral inequality and extensions. Coll. Math. 24(1), 87-91 (2008)

24. Xin, D, Yang, B: A basic Hilbert-type inequality. J. Math. 30(3), 554-560 (2010)

25. Hu, K: A few important inequalities. J. Jianxi Teach. Coll. Nat. Sci. 3(1), 1-4 (1979)

26. Pachpatte, BG: On some new inequalities similar to Hilbert's inequality. J. Math. Anal. Appl. 226, 166-179 (1998)

27. Zhao, C, Debnath, L: Some new type Hilbert integral inequalities. J. Math. Anal. Appl. 262, 411-418 (2001)

28. Lu, Z: Some new inverse type Hilbert-Pachpatte inequalities. Tamkang J. Math. 34(2), 155-161 (2003)

29. He, B, Li, Y: On several new inequalities close to Hilbert-Pachpatte's inequality. J. Inequal. Pure Appl. Math. 7(4), Article 154 (2006)

30. Pachpatte, BG: Mathematical Inequalities. Elsevier, Amsterdam (2005)

31. Yang, B: On Hilbert's integral inequality. J. Math. Anal. Appl. 220, 778-785 (1998)

32. Kuang, J: On new extension of Hilbert's integral inequality. J. Math. Anal. Appl. 235, 608-614 (1999)

33. Kuang, J: New progress in inequality study in China. J. Beijing Union Univ. Nat. Sci. 19(1), 29-37 (2005)

34. Gao, M: On the Hilbert inequality. J. Anal. Appl. 18(4), 1117-1122 (1999)

35. Gao, M, Hsu, L: A survey of various refinements and generalizations of Hilbert's inequalities. J. Math. Res. Expo. 25(2), 227-243 (2005)

36. Zhang, KW: A bilinear inequality. J. Math. Anal. Appl. 271, 288-296 (2002)

37. Hsu, L, Wang, Y: A refinement of Hilbert's double series theorem. J. Math. Res. Expo. 11(1), 143-144 (1991)

38. Gao, M: A note on Hilbert double series theorem. Hunan Ann. Math. 12(1-2), 143-147 (1992)

39. Xu, L, Guo, Y: Note on Hardy-Riesz's extension of Hilbert's inequality. Chin. Q. J. Math. 6(1), 75-77 (1991)

40. Yang, B, Gao, M: On a best value of Hardy-Hilbert's inequality. Adv. Math. 26(2), 159-164 (1997)

41. Gao, M, Yang, B: On the extended Hilbert's inequality. Proc. Am. Math. Soc. 126(3), 751-759 (1998)

42. Yang, B, Debnath, L: On new strengthened Hardy-Hilbert's inequality. Int. J. Math. Math. Sci. 21(2), $403-408$ (1998)

43. Yang, B: A refinement of Hilbert's inequality. Huanghuai J. 13(2), 47-51 (1997)

44. Yang, B: On a strengthened version of the more accurate Hardy-Hilbert's inequality. Acta Math. Sin. 42(6), 1103-1110 (1999)

45. Yang, B, Debnath, L: A strengthened Hardy-Hilbert's inequality. Proc. Jangjeon Math. Soc. 6(2), 119-124 (2003)

46. Yang, B: A note on Hilbert's integral inequalities. Chin. Q. J. Math. 13(4), 83-86 (1998)

47. Wang, Z, Guo, D: Introduction to Special Functions. Science Press, Beijing (1979)

48. Yang, B: A general Hardy-Hilbert's integral inequality with a best value. Chin. Ann. Math., Ser. A 21(4), 401-408 (2000)

49. Yang, B, Debnath, L: On the extended Hardy-Hilbert's inequality. J. Math. Anal. Appl. 272, 187-199 (2002)

50. Yang, B, Debnath, L: On a new generalization of Hardy-Hilbert's inequality. J. Math. Anal. Appl. 233, 484-497 (1999)

51. Yang, B: On a generalization of Hilbert's double series theorem. J. Nanjing Univ. Math. Biq. 18(1), 145-151 (2001)

52. Yang, B: On a general Hardy-Hilbert's inequality. Chin. Ann. Math., Ser. A 23(2), 247-254 (2002)

53. Yang, B: A dual Hardy-Hilbert's inequality and generalizations. Adv. Math. 35(1), 102-108 (2006)

54. Yang, B: On new extensions of Hilbert's inequality. Acta Math. Hung. 104(4), 291-299 (2004)

55. Yang, B: On a new inequality similar to Hardy-Hilbert's inequality. Math. Inequal. Appl. 6(1), 37-44 (2003)

56. Yang, B: Best generalization of Hilbert's type of inequality. J. Jilin Univ. Sci. Ed. 42(1), 30-34 (2004)

57. Yang, B: On a generalization of the Hilbert's type inequality and its applications. Chin. J. Eng. Math. 21(5), 821-824 (2004)

58. Yang, B: Generalization of the Hilbert's type inequality with best constant factor and its applications. J. Math. Res. Expo. 25(2), 341-346 (2005)

59. Yang, B: On Mulholland's integral inequality. Soochow J. Math. 31(4), 573-580 (2005)

60. Yang, B: A new Hilbert-type inequality. Bull. Belg. Math. Soc. Simon Stevin 13, 479-487 (2006)

61. Wang, W, Yang, B: A strengthened Hardy-Hilbert's type inequality. Aust. J. Math. Anal. Appl. 3(2), Article 17 (2006)

62. Hong, Y: All-side generalization about Hardy-Hilbert integral inequalities. Acta Math. Sin. 44(4), $619-626$ (2001)

63. He, L, Yu, J, Gao, M: An extension of Hilbert's integral inequality. J. Shaoguan Univ. Nat. Sci. 23(3), 25-30 (2002)

64. Yang, B: On a multiple Hardy-Hilbert's integral inequality. Chin. Ann. Math., Ser. A 24(6), 743-750 (2003)

65. Yang, B, Rassias, TM: On the way of weight coefficient and research for Hilbert-type inequalities. Math. Inequal. Appl. 6(4), 625-658 (2003)

66. Yang, B: On the way of weight function and research for Hilbert's type integral inequalities. J. Guangdong Educ. Inst. 25(3), 1-6 (2005)

67. Sulaiman, W: On Hardy-Hilbert's integral inequality. J. Inequal. Pure Appl. Math. 5(2), Article 25 (2004)

68. Brnetic, I, Pecaric, J: Generalization of Hilbert's integral inequality. Math. Inequal. Appl. 7(2), 199-205 (2004)

69. Krnić, M, Gao, M, Pečarić, J, Gao, X: On the best constant in Hilbert's inequality. Math. Inequal. Appl. 8(2), 317-329 (2005)

70. Brnet, I, Krnić, M, Pečarić, J: Multiple Hilbert and Hardy-Hilbert inequalities with non-conjugate parameters. Bull. Aust. Math. Soc. 71, 447-457 (2005)

71. Krnić, M, Pečarić, J: General Hilbert's and Hardy's inequalities. Math. Inequal. Appl. 8(1), 29-51 (2005) 
72. Sulaiman, W: New ideas on Hardy-Hilbert's integral inequality (I). Panam. Math. J. 15(2), 95-100 (2005)

73. Salem, SR: Some new Hilbert type inequalities. Kyungpook Math. J. 46, 19-29 (2006)

74. Laith, EA: On some extensions of Hardy-Hilbert's inequality and applications. J. Inequal. Appl. 2008, Article ID $546828(2008)$

75. Jia, W, Gao, M, Debnath, L: Some new improvement of the Hardy-Hilbert inequality with applications. Int. J. Pure Appl. Math. 11(1), 21-28 (2004)

76. Lu, Z: On new generalizations of Hilbert's inequalities. Tamkang J. Math. 35(1), 77-86 (2004)

77. Xie, H, Lu, Z: Discrete Hardy-Hilbert's inequalities in $\mathbf{R}^{n}$. Northeast. Math. J. 21(1), 87-94 (2005)

78. Gao, M: A new Hardy-Hilbert's type inequality for double series and its applications. Aust. J. Math. Anal. Appl. 3(1), Article 13 (2005)

79. He, L, Gao, M, Jia, W: On a new strengthened Hardy-Hilbert's inequality. J. Math. Res. Expo. 26(2), 276-282 (2006)

80. He, L, Jia, W, Gao, M: A Hardy-Hilbert's type inequality with gamma function and its applications. Integral Transforms Spec. Funct. 17(5), 355-363 (2006)

81. Jia, W, Gao, M, Gao, X: On an extension of the Hardy-Hilbert theorem. Studia Sci. Math. Hung. 42(1), 21-35 (2005)

82. Gao, M, Jia, W, Gao, X: On an improvement of Hardy-Hilbert's inequality. J. Math. 26(6), 647-651 (2006)

83. Sun, B: Best generalization of a Hilbert type inequality. J. Inequal. Pure Appl. Math. 7(3), Article 113 (2006)

84. Wang, W, Xin, D: On a new strengthened version of a Hardy-Hilbert type inequality and applications. J. Inequal. Pure Appl. Math. 7(5), Article 180 (2006)

85. Xu, J: Hardy-Hilbert's inequalities with two parameters. Adv. Math. 36(2), 189-198 (2007)

86. Chen, Z, Xu, J: New extensions of Hilbert's inequality with multiple parameters. Acta Math. Hung. 117(4), 383-400 (2007)

87. Yang, B: On an extension of Hilbert's integral inequality with some parameters. Aust. J. Math. Anal. Appl. 1(1), Article $11(2004)$

88. Yang, B, Brnetić, I, Krnić, M, Pečarić, J: Generalization of Hilbert and Hardy-Hilbert integral inequalities. Math. Inequal. Appl. 8(2), 259-272 (2005)

89. Hong, Y: On multiple Hardy-Hilbert integral inequalities with some parameters. J. Inequal. Appl. 2006, Article ID 94960 (2006)

90. Hong, Y: On Hardy-Hilbert integral inequalities with some parameters. J. Inequal. Pure Appl. Math. 6(4), Article 92 (2005)

91. Zhong, W, Yang, B: On a multiple Hilbert-type integral inequality with the symmetric kernel. J. Inequal. Appl. 2007, Article ID 27962 (2007)

92. Yang, B, Krnić, M: On the norm of a multi-dimensional Hilbert-type operator. Sarajevo J. Math. 7(20), 223-243 (2011)

93. Krnić, M, Pečarić, J, Vuković, P: On some higher-dimensional Hilbert's and Hardy-Hilbert's type integral inequalities with parameters. Math. Inequal. Appl. 11, 701-716 (2008)

94. Krnić, M, Vuković, P: On a multidimensional version of the Hilbert-type inequality. Anal. Math. 38, 291-303 (2012)

95. Rassias, MT, Yang, B: On a multidimensional half-discrete Hilbert-type inequality related to the hyperbolic cotangent function. Appl. Math. Comput. 242, 800-813 (2014)

96. Yang, B: On best extensions of Hardy-Hilbert's inequality with two parameters. J. Inequal. Pure Appl. Math. 6(3) Article 81 (2005)

97. Das, N, Sahoo, S: A generalization of Hardy-Hilbert's inequality for non-homogeneous kernel. Bul. Acad. Ştiinţe Repub. Mold. Mat. 67(3), 29-44 (2011)

98. Krnić, M, Pečarić, J, Vuković, P: Discrete Hilbert-type inequalities with general homogeneous kernels. Rend. Circ. Mat. Palermo 60(1-2), 161-171 (2011)

99. Adiyasuren, $\vee$, Batbold, T: On a relation between the Hardy-Hilbert and Gabriel inequalities. In: Rassias, TM (ed.) Handbook of Functional Equations: Functional Inequalities. Springer, Berlin (2014)

100. Yang, B: A reverse of the Hardy-Hilbert's type inequality. J. Southwest China Norm. Univ. Nat. Sci. 30(6), 1012-1015 (2005)

101. Yang, B: A reverse Hardy-Hilbert's integral inequality. J. Jilin Univ. Sci. Ed. 42(4), 489-493 (2004)

102. Yang, B: On a reverse of Hardy-Hilbert's integral inequality. Pure Appl. Math. 22(3), 312-317 (2006)

103. Yang, B: On an extended Hardy-Hilbert's inequality and some reversed form. Int. Math. Forum 1(39), 1905-1912 (2006)

104. Yang, B: A reverse of the Hardy-Hilbert's inequality. Math. Pract. Theory 36(11), 207-212 (2006)

105. Yang, B: On a reverse of a Hardy-Hilbert type inequality. J. Inequal. Pure Appl. Math. 7(3), Article 115 (2006)

106. Xi, G: A reverse Hardy-Hilbert-type inequality. J. Inequal. Appl. 2007, Article ID 79758 (2007)

107. Yang, B: On a relation to Hardy-Hilbert's inequality and Mulholland's inequality. Acta Math. Sin. 49(3), $559-566$ (2006)

108. Xin, D: Best generalization of Hardy-Hilbert's inequality with multi-parameters. J. Inequal. Pure Appl. Math. 7(4), Article 153 (2006)

109. Zhong, W, Yang, B: A best extension of Hilbert inequality involving several parameters. J. Jinan Univ. Nat. Sci. 28(1), 20-23 (2007)

110. Zhong, W, Yang, B: A reverse Hilbert's type integral inequality with some parameters and the equivalent forms. Pure Appl. Math. 24(2), 401-407 (2008)

111. Yang, B: A new Hilbert-type inequality. J. Shanghai Univ. Nat. Sci. 13(3), 274-278 (2007)

112. Yang, B: A bilinear inequality with a homogeneous kernel of -2-order. J. Xiamen Univ. Nat. Sci. 45(6), 752-755 (2006)

113. Yang, B: A Hilbert-type integral inequality with the kernel of -3-order homogeneous. J. Yunnam Univ. 30(4), 325-330 (2008)

114. Xie, Z: A new Hilbert-type inequality with the kernel of 3-homogeneous. J. Jilin Univ. Sci. Ed. 45(3), 369-373 (2007)

115. Xie, Z, Zheng, Z: A Hilbert-type inequality with parameters. J. Xiangtan Univ. Nat. Sci. 29(3), 24-28 (2007)

116. Xie, Z, Zheng, Z: A Hilbert-type integral inequality whose kernel is a homogeneous form of degree -3. J. Math. Anal. Appl. 339, 324-331 (2007)

117. Xie, Z, Zheng, Z: A new Hilbert-type integral inequality and its reverse. Soochow J. Math. 33(4), 751-759 (2007)

118. Li, Y, He, B: On inequalities of Hilbert's type. Bull. Aust. Math. Soc. 76, 1-13 (2007)

119. He, B, Qian, Y, Li, Y: On analogues of the Hilbert's inequality. Commun. Math. Anal. 4(2), 47-53 (2008) 
120. Yang, B: On a Hilbert-type inequality with the homogeneous kernel of -3-order. J. Guangdong Educ. Inst. 27(5), 1-5 (2007)

121. Yang, B: A mixed Hilbert-type inequality with a best constant factor. Int. J. Pure Appl. Math. 20(3), 319-328 (2005)

122. Yang, B: A half-discrete Hilbert's inequality. J. Guangdong Univ. Educ. 31(3), 1-7 (2011)

123. Zhong, W: A mixed Hilbert-type inequality and its equivalent forms. J. Guangdong Univ. Educ. 31(5), 18-22 (2011)

124. Rassias, MT, Yang, B: On half-discrete Hilbert's inequality. Appl. Math. Comput. 220, 75-93 (2013)

125. Yang, B, Chen, Q: A half-discrete Hilbert-type inequality with a homogeneous kernel and an extension. J. Inequal. Appl. 2011, 124 (2011)

126. Yang, B: A half-discrete Hilbert-type inequality with a non-homogeneous kernel and two variables. Mediterr. J. Math. 10, 677-692 (2013)

127. Yang, B: On the norm of an integral operator and applications. J. Math. Anal. Appl. 321, 182-192 (2006)

128. Yang, B: On the norm of a self-adjoint operator and a new bilinear integral inequality. Acta Math. Sin. Engl. Ser. 23(7), 1311-1316 (2007)

129. Yang, B: On the norm of a certain self-adjoint integral operator and applications to bilinear integral inequalities. Taiwan. J. Math. 12(2), 315-324 (2008)

130. Yang, B: On the norm of a Hilbert's type linear operator and applications. J. Math. Anal. Appl. 325, $529-541$ (2007)

131. Yang, B: On the norm of a self-adjoint operator and applications to Hilbert's type inequalities. Bull. Belg. Math. Soc. Simon Stevin 13, 577-584 (2006)

132. Yang, B: On a Hilbert-type operator with a symmetric homogeneous kernel of -1-order and applications. J. Inequal. Appl. 2007, Article ID 47812 (2007)

133. Yang, B: On the norm of a linear operator and its applications. Indian J. Pure Appl. Math. 39(3), 237-250 (2008)

134. Bényi, A, Oh, C: Best constants for certain multilinear integral operator. J. Inequal. Appl. 2006, Article ID 28582 (2006)

135. Yang, B: A survey of the study of Hilbert-type inequalities with parameters. Adv. Math. 38(3), $257-268$ (2009)

136. Yang, B: On a Hilbert-type operator with a class of homogeneous kernels. J. Inequal. Appl. 2009, Article ID 572176 (2009)

137. Huang, Q, Yang, B: On a multiple Hilbert-type integral operator and applications. J. Inequal. Appl. 2009, Article ID 192197 (2009)

138. Yang, B, Rassias, TM: On a Hilbert-type integral inequality in the subinterval and its operator expression. Banach J. Math. Anal. 4(2), 100-110 (2010)

139. Liu, X, Yang, B: On a new Hilbert-Hardy-type integral operator and applications. J. Inequal. Appl. 2010, Article ID $812636(2010)$

140. Yang, B: A new Hilbert-type operator and applications. Publ. Math. (Debr.) 76(1-2), 147-156 (2010)

141. Yang, B, Krnić, M: Hilbert-type inequalities and related operators with homogeneous kernel of degree 0. Math. Inequal. Appl. 13(4), 817-839 (2010)

142. Adiyasuren, $\vee$, Batbold, T, Krnić, M: On several new Hilbert-type inequalities involving means operators. Acta Math. Sin. Engl. Ser. 29(8), 1493-1514 (2013)

143. Liu, X, Yang, B: On a new Hilbert-Hardy-type integral operator and applications. J. Inequal. Appl. 2010, Article ID $812636(2010)$

144. Adiyasuren, V, Batbold, T, Krnić, M: Half-discrete Hilbert-type inequalities with mean operators, the best constants, and applications. Appl. Math. Comput. 231, 148-159 (2014)

145. Wang, A, Yang, B: A new Hilbert-type integral inequality in the whole plane with the non-homogeneous kernel. J. Inequal. Appl. 2011, 123 (2011)

146. Yang, B: On the Norm of Operator and Hilbert-Type Inequalities. Science Press, Beijing (2009)

147. Yang, B: Hilbert-Type Integral Inequalities. Bentham Science Publishers Ltd., Sharjah (2009)

148. Yang, B: Discrete Hilbert-Type Inequalities. Bentham Science Publishers Ltd., Sharjah (2011)

149. Yang, B: Two Kinds of Multiple Half-Discrete Hilbert-Type Inequalities. Lambert Academic Publishing, Saarbrücken (2012)

150. Yang, B: Topics on Half-Discrete Hilbert-Type Inequalities. Lambert Academic Publishing, Saarbrücken (2013)

151. Yang, B: Hilbert-type integral operators: norms and inequalities. In: Pardalos, PM, Georgiev, PG, Srivastava, HM (eds.) Nonlinear Analysis: Stability, Approximation, and Inequalities. Springer, Berlin (2012)

152. Rassias, MT, Yang, B: A multidimensional Hilbert-type integral inequalities related to the Riemann zeta function. In: Daras, NJ (ed.) Applications of Mathematics and Informatics in Science and Engineering. Springer, Berlin (2014)

153. Yang, B: On Hilbert-type integral inequalities and their operator expressions. J. Guangdong Univ. Educ. 33(5), 1-17 (2013)

154. Yang, B: Multidimensional discrete Hilbert-type inequalities, operators and compositions. In: Milovanović, GV, Rassias, MT (eds.) Analytic Number Theory, Approximation Theory, and Special Functions. Springer, Berlin (2014)

155. Yang, B, Debnath, L: Half-Discrete Hilbert-Type Inequalities. World Scientific, Singapore (2014)

156. Yang, B: Half-discrete Hilbert-type inequalities, operators and compositions. In: Rassias, TM (ed.) Handbook of Functional Equations: Functional Inequalities. Springer, Berlin (2014)

157. Yang, B: A basic Hilbert-type integral inequality with the homogeneous kernel of -1-degree and extensions. J. Guangdong Educ. Inst. 28(3), 1-10 (2008)

158. Yang, B: A new Hilbert-type integral inequality and its generalization. J. Jilin Univ. Sci. Ed. 43(5), 580-584 (2005)

159. Yang, B, Liang, H: A new Hilbert-type integral inequality with a parameter. J. Henan Univ. Nat. Sci. 35(4), 4-8 (2005) 